\title{
Histone methyltransferase SETD2 modulates alternative splicing to inhibit intestinal tumorigenesis
}

\author{
Huairui Yuan, ${ }^{1} \mathrm{Ni}$ Li, ${ }^{1}$ Da Fu, ${ }^{2}$ Jiale Ren, ${ }^{1}$ Jingyi Hui, ${ }^{3}$ Junjie Peng, ${ }^{4}$ Yongfeng Liu, ${ }^{1}$ Tong Qiu, ${ }^{5}$ Min Jiang, ${ }^{1}$ Qiang Pan, ${ }^{1}$ Ying Han, ${ }^{1}$ \\ Xiaoming Wang, ${ }^{6}$ Qintong Li, ${ }^{5}$ and Jun Q ${ }^{1}{ }^{1}$

\begin{abstract}
'The Key Laboratory of Stem Cell Biology, CAS Center for Excellence in Molecular Cell Science, Institute of Health Sciences, Shanghai Institutes for Biological Sciences, Chinese Academy of Sciences/ Shanghai Jiao Tong University School of Medicine, University of Chinese Academy of Sciences, Shanghai, China. '2entral Laboratory for Medical Research, Shanghai Tenth People's Hospital, Tongji University School of Medicine, Shanghai, China. 'State Key Laboratory of Molecular Biology, CAS Center for Excellence in Molecular Cell Science, Shanghai Institute of Biochemistry and Cell Biology, Chinese Academy of Sciences, University of Chinese Academy of Sciences, Shanghai, China. ${ }^{4}$ Department of Colorectal Surgery, Fudan University Shanghai Cancer Center, Shanghai, China. ${ }^{5}$ Department of Obstetrics, Gynecology and Pediatrics, West China Second University Hospital, Key Laboratory of Birth Defects and Related Diseases of Women and Children, Ministry of Education, Sichuan University, Chengdu, China. ${ }^{6}$ Department of Immunology, Nanjing Medical University, Nanjing, China.
\end{abstract}

\begin{abstract}
The histone H3K36 methyltransferase SETD2 is frequently mutated or deleted in a variety of human tumors. Nevertheless, the role of SETD2 loss in oncogenesis remains largely undefined. Here, we found that SETD2 counteracts Wnt signaling and its inactivation promotes intestinal tumorigenesis in mouse models of colorectal cancer (CRC). SETD2 was not required for intestinal homeostasis under steady state; however, upon irradiation, genetic inactivation of Setd2 in mouse intestinal epithelium facilitated the self-renewal of intestinal stem/progenitor cells as well as tissue regeneration. Furthermore, depletion of SETD2 enhanced the susceptibility to tumorigenesis in the context of dysregulated Wnt signaling. Mechanistic characterizations indicated that SETD2 downregulation affects the alternative splicing of a subset of genes implicated in tumorigenesis. Importantly, we uncovered that SETD2 ablation reduces intron retention of dishevelled segment polarity protein 2 (DVL2) pre-mRNA, which would otherwise be degraded by nonsense-mediated decay, thereby augmenting Wnt signaling. The signaling cascades mediated by SETD2 were further substantiated by a CRC patient cohort analysis. Together, our studies highlight SETD2 as an integral regulator of Wnt signaling through epigenetic regulation of RNA processing during tissue regeneration and tumorigenesis.
\end{abstract}

\section{Introduction}

Epigenetic regulation plays key roles in tumor evolutions. Among these factors, histone methyltransferases (HMTs) constitute appealing targets for disease intervention because they are frequently dysregulated in a spectrum of human tumors and their enzymatic activity could be therapeutically manipulated (1). Nevertheless, it remains unclear in many cases whether alterations in histone modifications are the driving force to foster tumor cell heterogeneity and neoplastic predisposition.

The interplay between active and repressive histone modifications governs gene expression. In general, histone $\mathrm{H} 3$ lysine 27 trimethylation (H3K27me3) leads to gene repression, whereas histone $\mathrm{H} 3$ lysine 4 trimethylation (H3K4me3) and $\mathrm{H} 3$ lysine 36 methylation (H3K36me2/me3) promote gene transcription (2). In mammalian cells, SETD2 is the major enzyme that catalyzes the trimethylation of lysine 36 on histone 3 (H3K36me3) $(3,4)$, a histone mark associated with actively transcribed regions $(2,5)$. Germline

Related Commentary: p. 3281

Authorship note: H. Yuan, N. Li, and D. Fu contributed equally to this work Conflict of interest: The authors have declared that no conflict of interest exists Submitted: March 30, 2017; Accepted: June 23, 2017.

Reference information: J Clin Invest. 2017;127(9):3375-3391.

https://doi.org/10.1172/JCI94292 inactivation of Setd2 in mice leads to vascular remodeling defects (4), indicating a nonredundant role of SETD2 during development. Cell culture-based studies demonstrate the defects in chromosome segregation, DNA repair, and reduced nucleotide pools in SETD2-depleted cells (6-8). Mechanistic characterizations reveal that SETD2-mediated H3K36me3 regulates the recruitment of and DNMT3b to ensure the fidelity of gene transcription initiation in embryonic stem (ES) cells (9). In addition, SETD2 and H3K36me3 are implicated in RNA splicing during gene transcription (10-13). SETD2-mediated H3K36me3 influences the splice site selection through the recruitment of MORF-related gene 15 (MRG15) and polypyrimidine tract binding protein 1 (PTB) (14). Consistent with its prominent role in maintenance of genomic integrity, SETD2 is frequently mutated or deleted in a variety of human tumors, most pronounced in clear cell renal cell carcinoma (ccRCC), but also in breast, glioma, gastrointestinal stromal tumors (GISTs), and leukemia (15-19). Nevertheless, the genetic characterization of SETD2 to determine its role in tumorigenesis and the signaling pathways coordinated remains largely undetermined.

Colorectal cancer (CRC), one of the most deadly cancers worldwide, arises from stepwise accumulation of genetic and epigenetic perturbations (20-22). As one of the most vigorously renewing tissues, intestine represents an excellent model for understanding the molecular mechanisms that modulate tissue homeostasis. Wnt/ $\beta$-catenin signaling activates intestinal stem cells (ISCs) to 
give rise to progenitor cells and, afterwards, replenish the intestinal epithelium (23). The threshold of Wnt signaling within the stem or progenitor cells is tightly regulated to maintain intestine homeostasis. Disruption of such tight regulation by inactivation of the tumor suppressor gene APC or activation of the oncogene CTNNB1 is frequently observed in the vast majority of human CRCs (22). Of note, Wnt target gene leucine-rich repeat-containing $G$ proteincoupled receptor 5 ( $\operatorname{Lgr} 5)$ is demonstrated as a specific proliferating stem cell marker, and lineage-tracing experiments indicate that Lgr5-positive cells can be the origin of intestinal adenomas in mice (24). During intestinal tumorigenesis, Wnt/ $\beta$-catenin signaling is essential for stimulating the propagations of the tumor cells partially bearing stem or progenitor cell characteristics. However, the Wnt pathway involves various feedback loops that balance the opposing processes of cell proliferation and differentiation $(25,26)$. Even in these Apc-mutated tumors, the population of cells sustaining stem or progenitor cell characteristics remains limited. Thus, beyond genetic mutations for Wnt signaling, additional layers of regulations exist to influence the full progression of CRC.

Here, we utilize genetically engineered mouse models (GEMs) and establish that SETD2 is a putative tumor suppressor gene in CRC. Mechanistic investigations indicate that SETD2 modulates the alternative splicing (AS) of the genes implicated in tumorigenesis. Interestingly, we show that SETD2 loss results in the accumulation of dishevelled segment polarity protein 2 (DVL2) mRNA through nonsense-mediated decay-coupled (NMD-coupled) AS regulation and consequently augments Wnt/ $\beta$-catenin signaling to affect intestinal self-renewal and differentiation. Thus, our results highlight that tumors lacking this methyltransferase exhibit a more aggressive disease and worse outcome with potentially therapeutic implications.

\section{Results}

SETD2 expression is downregulated in human CRC. To explore a possible role of SETD2 in CRC, we first assessed SETD2 expression in humans with CRC. As shown in Figure 1A, quantitative reverse-transcriptase PCR (RT-qPCR) analysis indicated that SETD2 mRNA was significantly reduced in tumors compared with their paired normal biopsies (Figure 1A). In addition, Western blotting analysis revealed that levels of SETD2 protein and H3K36me3 were both decreased in the tumor lysates as compared with the normal counterparts (Figure 1B). To substantiate this observation, we extended the analysis to a larger patient cohort comprising 168 tumor specimens and 48 normal biopsies (the clinical information was summarized in Supplemental Table 1; supplemental material available online with this article; https://doi.org/10.1172/JCI94292DS1). Immunochemistry analyses revealed a significantly lower SETD2 protein level in colorectal tumors (mean $=3.97$, median $=4$ ) in comparison with normal colons (mean $=5.65$, median $=6$ ). Quantification showed that approximately $65 \%$ of normal tissues displayed intermediate to intense nuclear SETD2 staining, whereas only around $35 \%$ of tumors exhibited such a pattern (Figure 1C). Correlation studies also indicated that SETD2 expression in tumor cells was inversely linked to the stages of disease progression (Figure 1D). More importantly, as judged by hazard ratios (HRs), SETD2 expression levels served as an independent predictor for risk stratification of overall survival and metastasis-free survival in patients (Figure 1E and Supplemental Table 1). In addition, multivariate Cox proportional-hazards regression analysis revealed that SETD2 also carried the independent predictive power to further enhance the prognostic accuracy of TNM status in CRC ( $\mathrm{T}$, size or direct extent of the primary tumor; $\mathrm{N}$, degree of spread to regional lymph node; $\mathrm{M}$, presence of distant metastasis) (Supplemental Table 1). Likewise, the clinical relevance of SETD2 was further supported by analysis of the public data sets. As shown by Kaplan-Meier plot, SETD2 mRNA was negatively associated with disease-relapsed free survival in 2 separate patient cohorts (Figure 1F). Together, these findings highlight SETD2 as a potential biomarker for CRC and suggest a causal role of SETD2 in colorectal tumorigenesis.

SETD2 reduction promotes tumorigenesis in cells. To investigate the role of SETD2 in colon cancer, we first generated SETD2depleted RKO and DLD1 cells using 2 shRNA constructs that display similar knockdown efficiency (Figure 2A). Reduced SETD2 facilitated the growth and migration of these cells as measured by MTT and Transwell assays, respectively (Figure 2, B and C). Next, we examined whether SETD2 is important for anchorageindependent growth and oncosphere formation in cells. As shown in Figure 2, D and E, reduced SETD2 expression in HCT116, RKO, and DLD1 cells markedly enhanced anchorage-independent growth and oncosphere formation. Quantitation results revealed there were 2- to 3-fold increases of colonial formation efficiency upon SETD2 silencing (Figure 2D). Although overexpression of SETD2 did not affect the growth and migration of HCT116 cells (Supplemental Figure 1), it impaired colony and oncosphere growth (Figure 2F). In contrast, a SETD2 mutant lacking the catalytical set domain did not exhibit such impact, suggesting that methyltransferase activity is required for its suppressive function. Together, our results indicate that SETD2 impairs CRC progress in CRC cells.

Setd2 deletion aggravates tumor malignance in an Apc ${ }^{\text {min/t }}$ mouse model. The above results prompted us to employ GEMs to investigate the role of SETD2 in CRC in vivo. To circumvent embryonic lethality, we first generated Setd2-floxed mice (Supplemental Figure 2A) and crossed them with Villin-Cre mice to delete Setd2 in intestinal epithelium $\left(\operatorname{Setd} 2^{f / f l}\right.$ Villin $^{\text {Cre/ }}$, hereafter referred as Setd2 $2^{\mathrm{SEC}}$ mice) (Figure 3, A and B, and Supplemental Figure 2A). Interestingly, though inactivation of Setd2 profoundly reduced H3K36me3 levels within intestinal epithelium (Figure $3, \mathrm{~A}$ and B), Setd $2^{\mathrm{SIEC}}$ mice were viable and fertile and exhibited no gross phenotypic abnormalities compared with their control littermates. Over a period of 10-month observations, no overt defects were seen in the structures of intestine and the crypt-villus axis in Setd2 $2^{\mathrm{IIEC}}$ mice (Figure $3 \mathrm{C}$ and Supplemental Figure 2B). In addition, by assessing the number of enterocytes and goblet cells (identified by alkaline phosphatase [ALP] and periodic acid-Schiff [PAS] staining), we determined there were no detectable differences in terms of cell lineage composition (Supplemental Figure 2B). This observation was further confirmed by RT-qPCR analysis. Though MUC2 expression (goblet cell) was decreased, there were no significant changes in the expression of other marker genes for the different cell lineages and stem cell populations in SETD2-deficient intestinal tissue compared with controls (Supplemental Figure 2C). Thus, Setd2 is largely dispensable for intestinal homeostasis under physiological states. 
A



E
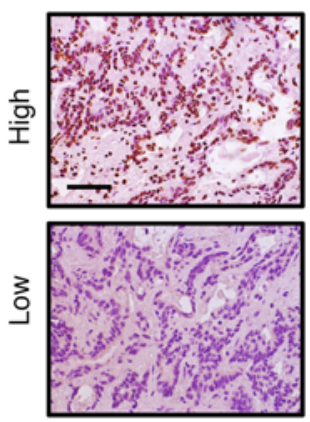

F

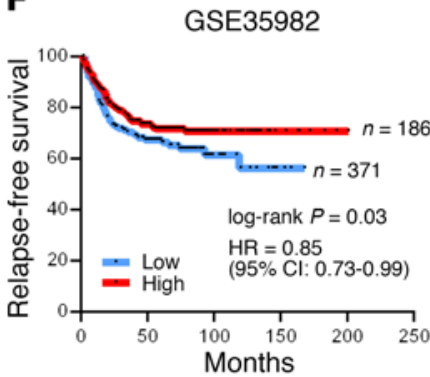

B
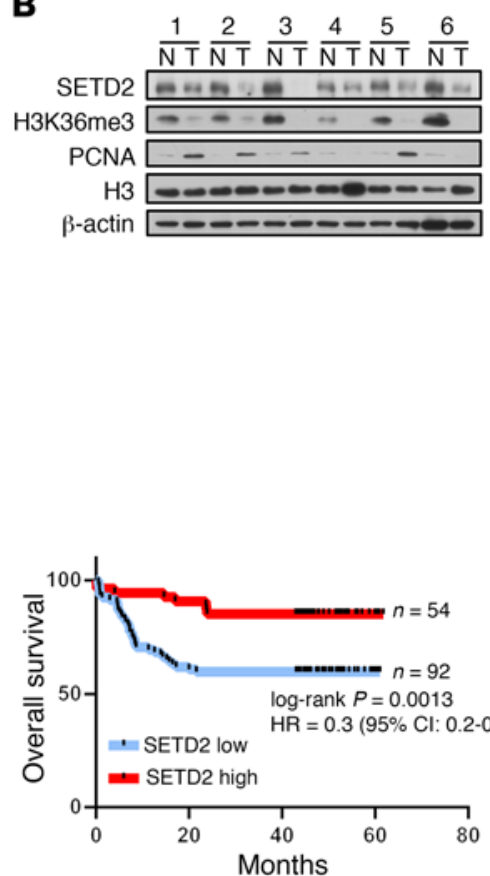

C
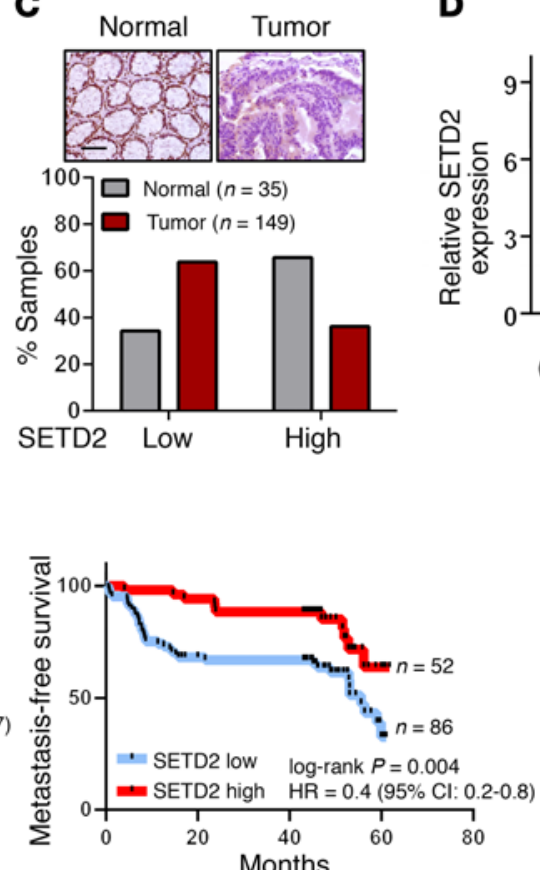

D

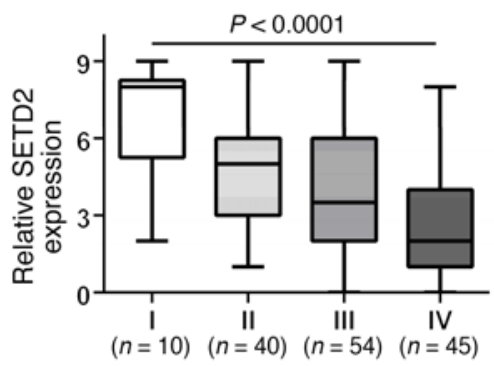

Figure 1. Clinical relevance of SETD2 expression in human CRC. (A) SETD2 mRNA levels in 30 matched tumor and paracarcinoma tissues (paired $t$ test, $P=0.0035$ ). (B) SETD2 and H3K36me3 protein levels in 6 pairs of random CRC samples. Blot images are derived from replicate samples run on parallel gels. N, adjacent normal specimens; T, matched tumor tissues. (C) Expression of SETD2 was assessed in tissue microarray. SETD2 staining indexes using a 10 -point quantification scale in normal colon counterparts $(n=35)$ and tumors $(n=149)$ are shown $\left(\chi^{2}\right.$ test, $\left.P=0.0015\right)$. (D) Boxed plot of SETD2 expression assessed by blinded IHC analyses of TMA at different clinical stages (Kruskal-Wallis test). (E) Kaplan-Meier plot of overall survival and metastasis-free survival of patients based on SETD2 expression levels. A log-rank test was used for statistical analysis. (F) Kaplan-Meier survival curves for disease relapse-free stratified by SETD2 expression levels using GEO data sets GSE35982 and GSE17538 ( $P$ values by log-rank test). Scale bars: $50 \mu$ m.

Mutation of the WT Apc allele $\left(\mathrm{Apc}^{\mathrm{min} /+}\right)$ results in widespread adenomas throughout the gastrointestinal tract in mice. We hypothesized that Setd2 deletion might aggravate tumorigenesis in $\mathrm{Apc}^{\mathrm{min} /+}$ mice. Thus, tumor progression of compound $\mathrm{Apc}^{\mathrm{min} /+}$; Setd2 $2^{\mathrm{IEC}}$ mice and the control $\mathrm{Apc}^{\mathrm{min} /+}$ group was analyzed over a period of 8 months. As shown by Kaplan-Meier survival plots, life span was much shorter in the compound mice, with a median survival of 105 days, compared with 205 days in $\mathrm{Apc}^{\mathrm{min} /+}$ mice $\left(P=8.66 \times 10^{-16}\right)$ (Figure 3D). Macroscopic dissections revealed a profound increase in the number of lesions and tumor loads within $\mathrm{Apc}^{\mathrm{min} /+}$; Setd2 $2^{\mathrm{IEC}}$ mice at 3 to 4 months of age (Figure $3 \mathrm{E}$ ). Of note, analyses of tumorsize distribution indicated that tumors in $\mathrm{Apc}^{\mathrm{min} /+}$; Setd2 ${ }^{\mathrm{\triangle IEC}}$ mice were larger in size compared with the lesions in $\mathrm{Apc}^{\mathrm{min} /{ }^{+}}$mice (Figure $3 \mathrm{~F}$ ), suggesting that Setd2 depletion promotes both tumor initiation and progression. H\&E staining further confirmed that there were significantly more polyps in the intestines of the compound mice (Figure 3G). At 3 to 4 months, subsets of the lesions arising from the compound mice displayed an adenomatous pattern invading the muscle layer, whereas most of the lesions in $\mathrm{Apc}^{\mathrm{min} /+}$ mice remained in the confined dysplastic stage (Figure 3G). Accordingly, the intestines of $\mathrm{Apc}^{\mathrm{min} /+}$; Setd2 ${ }^{\mathrm{AEC}}$ mice exhibited higher proliferative rates

Table 1. Summary of altered AS genes upon Setd2 knockout in Apc $^{\mathrm{min} /+}$ mice

$\begin{array}{lcc}\text { Type of AS events } & \text { Cene number } & \text { Total (\%) } \\ 3 \text { SSS } & 40 & 5.6 \% \\ \text { 5SS } & 33 & 4.6 \% \\ \text { RI } & 279 & 39.2 \% \\ \text { SE } & 198 & 27.8 \% \\ \text { MXE } & 161 & 22.6 \%\end{array}$



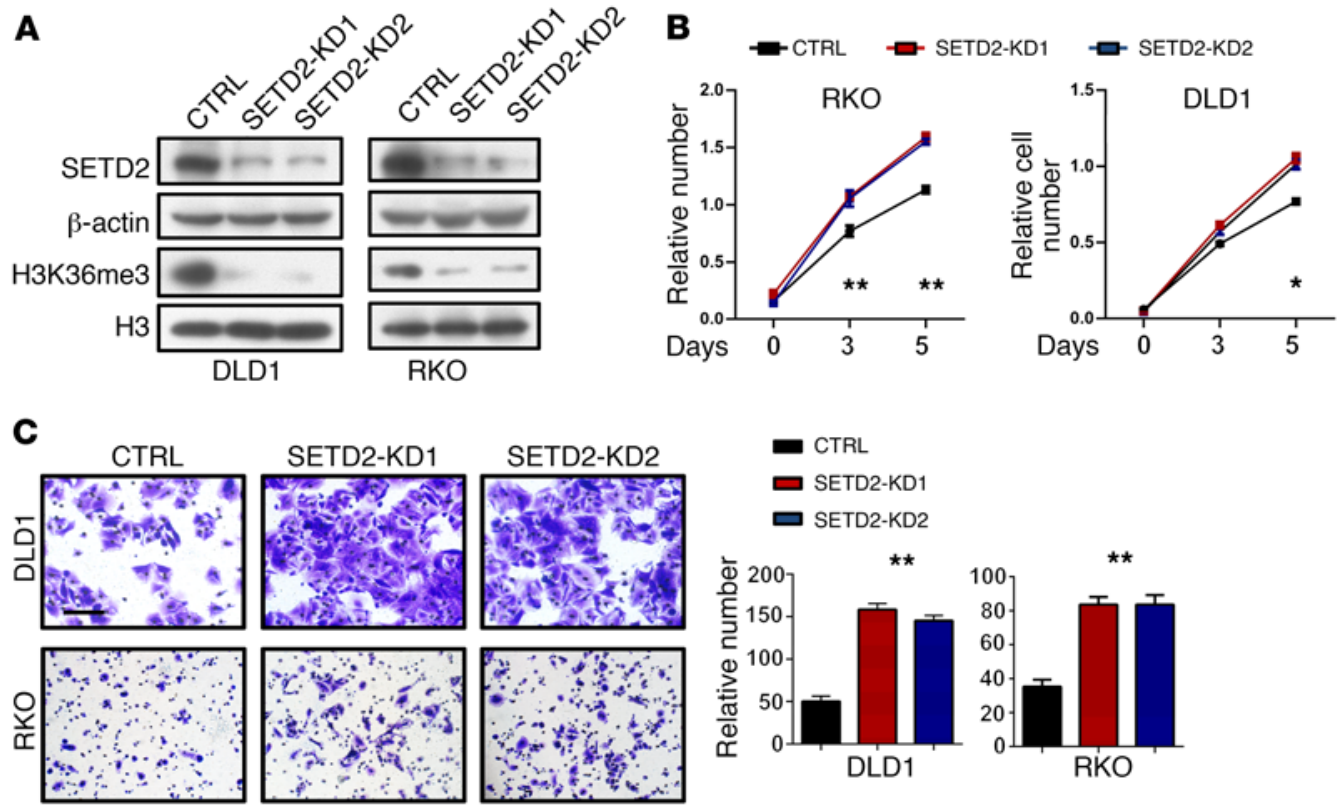

D
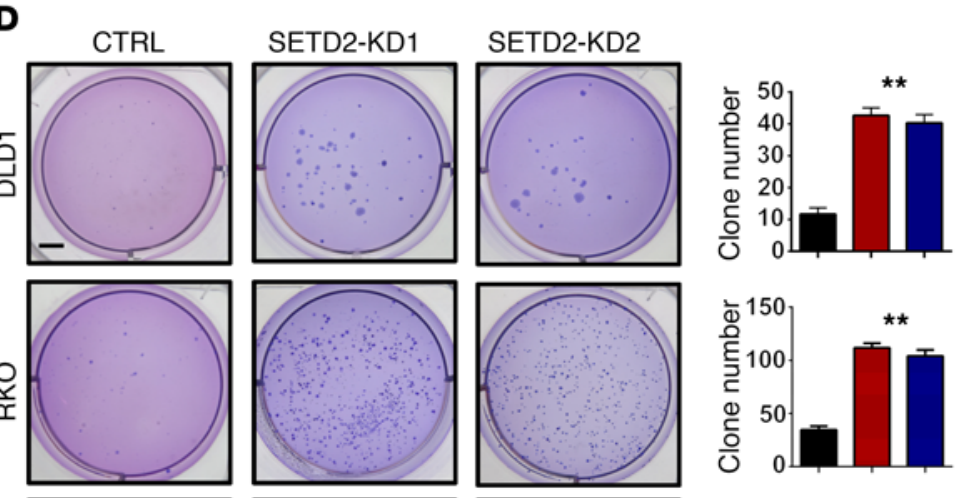

E
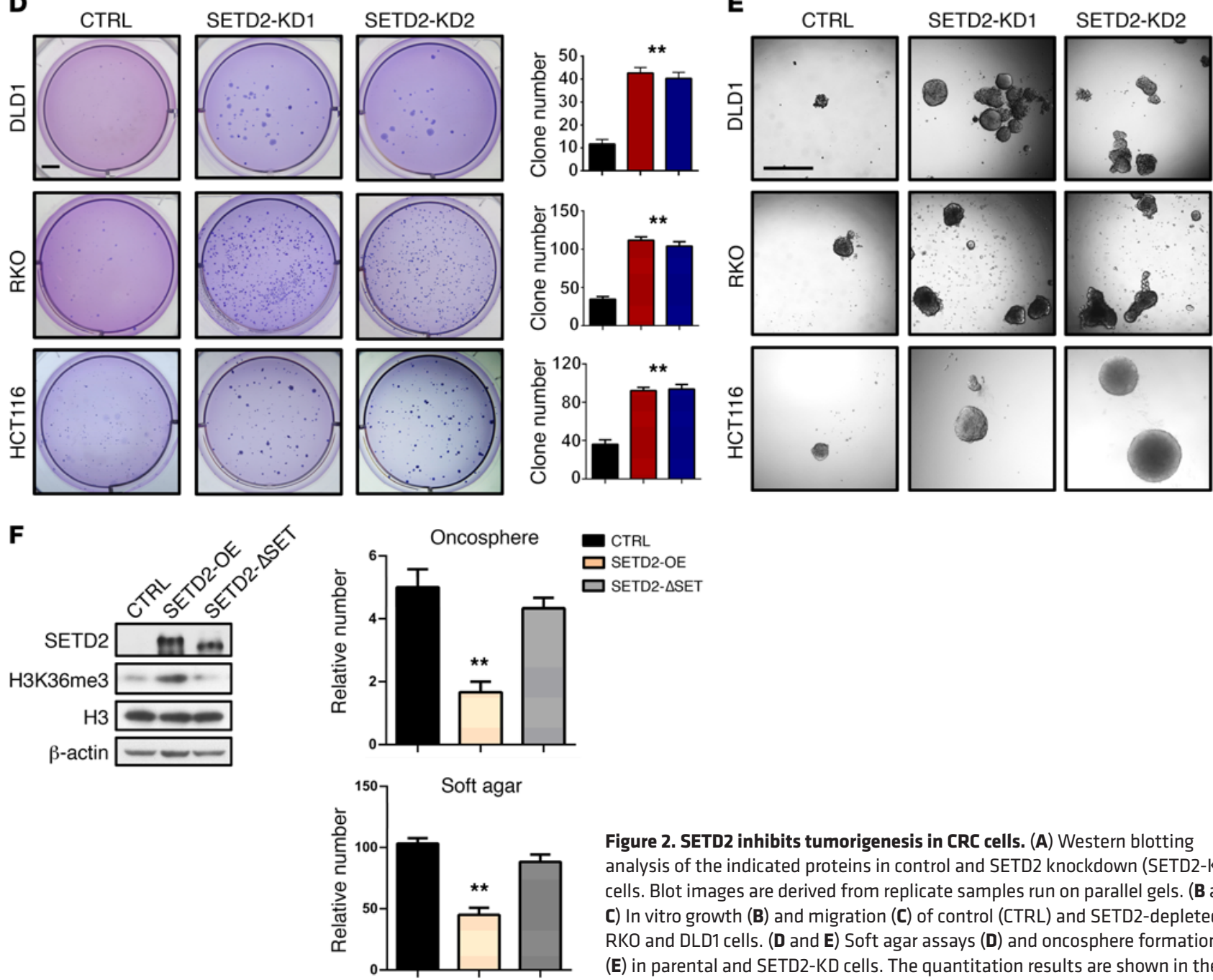

Figure 2. SETD2 inhibits tumorigenesis in CRC cells. (A) Western blotting analysis of the indicated proteins in control and SETD2 knockdown (SETD2-KD) cells. Blot images are derived from replicate samples run on parallel gels. (B and C) In vitro growth (B) and migration (C) of control (CTRL) and SETD2-depleted RKO and DLD1 cells. ( $\mathbf{D}$ and $\mathbf{E}$ ) Soft agar assays (D) and oncosphere formations (E) in parental and SETD2-KD cells. The quantitation results are shown in the right panels. (F) The levels of WT SETD2 or SETD2- $\Delta$ Set overexpression (left panel) and quantitation results of anchorage-independent growth and oncosphere formation (right panel) in HCT116 cells. Statistical comparisons in B-D, and $\mathbf{F}$ were made using a 2-tailed Student's $t$ test ${ }^{*} P<0.05$; $^{* *} P<0.01$. Scale bars: $400 \mathrm{~mm}$ (C); $5 \mathrm{~mm}$ (D); $1 \mathrm{~mm}$ (E). 
and less apoptosis, indicated by phospho-H3 and cleaved caspase 3 staining (Figure 3H and Supplemental Figure 3A). The observation that SETD2 deficiency promotes intestinal proliferation and prevents apoptosis was further strengthened by Western blotting analysis. As shown in Figure 3I, there were significant increases of proliferating cell nuclear antigen (PCNA) levels and reductions of cleaved caspase 3 signals in the intestine lysates of Apc $\mathrm{Cin}^{\mathrm{mi}+}$; Setd2 ${ }^{\mathrm{AEC}}$ mice. Together, these results demonstrate that SETD2 deficiency accelerates adenoma development in $\mathrm{Apc}^{\mathrm{min} /+}$ mice and support the notion that SETD2 is a tumor suppressor in the gut.

Setd2 deletion stimulates Wnt-dependent transformation and stemness programs. To get an insight into the mechanism of how Setd2 ablation promotes CRC, we performed gene expression analysis using the intestinal tissues isolated from $\mathrm{Apc}^{\mathrm{min} /+}$ and $\mathrm{Apc}^{\mathrm{min} /+}$; Setd $2^{\triangle I E C}$ mice. We utilized morphologically normal tissues without the polyps from 6- to 8-week-old mice. At this time point, $\mathrm{Apc}^{\mathrm{min} /+}$; Setd $2^{\triangle I E C}$ mice exhibited histology similar to that of the control littermates; therefore, the genes exhibiting altered expressions might be more directly related to earlier events influenced by SETD2. Ingenuity Pathway Analysis (IPA) indicated that the most prominently altered disease processes were associated with cancer and gastrointestinal disease (Supplemental Figure 3B). Similarly, cell proliferation and growth and cell death and survival were enriched cellular functions in the absence of SETD2 (Supplemental Figure 3B). To better understand SETD2-mediated signal circuits, we performed gene set enrichment analysis (GSEA) and observed that SETD2 loss significantly enriched the gene concepts linked to stem and progenitor signature, including stem cell and proliferation signaling, whereas terminal differentiation signaling was compromised in the absence of SETD2 (Figure 4A). These results were further exemplified by PAS, ALP staining, and anti-CD44 immunostaining. We found that the proportions of goblet cells (PAS positive) and enterocytes (ALP positive) were markedly reduced within the tumors of $A \mathrm{Ac}^{\mathrm{min} /+}$; Setd $2^{\mathrm{AICC}}$ mice, whereas the tumors lacking SETD2 were more heavily populated with CD44-positive progenitor cells (Figure 4B).

Interestingly, GSEA revealed that SETD2-mediated genes resemble the Wnt-responsive gene signature. As indicated in Figure 4A, genes upregulated by Wnt stimulation were enriched in the set of genes that were upregulated in response to SETD2 depletion, suggesting an inverse relationship between SETD2 and Wnt pathway activity. As summarized by heat map, RT-qPCR analysis verified that expressions of Wnt-responsive genes such as Axin2, Fzd7, Apcdd1, Sox9, and Myc were upregulated in SETD2-depleted intestines (Figure $4 \mathrm{C}$ ). To determine whether this regulation is cell autonomous, we depleted SETD2 in HCT116 cells and found that SETD2 silencing sensitized cells to Wnt3a stimulation, as reflected by the significantly increased expression of Wnt-induced target genes (Figure 4D). In agreement with the enhanced Wnt signaling in the absence of SETD2, RNAscope in situ hybridization assay detected a significantly increased number of Olfm4-positive stem cells within the crypts of Apc ${ }^{\mathrm{min} /+}$; Setd2 ${ }^{\mathrm{AIEC}}$ mice (Figure $4 \mathrm{E}$ ). Likewise, immunostaining of CD44 and SOX9 revealed the expansion of transamplified or progenitor zones within intestinal crypts of $\mathrm{Apc}^{\mathrm{min} /+}$; Setd2 $2^{\mathrm{AEC}}$ mice compared with those of $\mathrm{Apc}^{\mathrm{min} /+}$ mice (Figure $4 \mathrm{~F}$ ). Similarly, Wnt-mediated Paneth cells were observed to be mislocalized away from the home of the crypt (Figure $4 \mathrm{G}$ ). In addition, we also mea- sured $\beta$-catenin accumulation in the small intestine. Compared with control mice, nuclear $\beta$-catenin staining was enhanced in the crypts of histologically normal intestines of $\mathrm{Apc}^{\mathrm{min} /+}$; Setd $2^{\triangle \mathrm{IEC}}$ mice, and this accumulation was more prominent in the tumors lacking SETD2 (Figure 4H). Together, these results indicate that SETD2 deficiency stimulates Wnt-induced transformation and stemness programs to promote the expansion of stem/progenitor cells.

SETD2 loss enhances intestinal regeneration and self-renewal. When damaged, the intestine requires high levels of Wnt/ $\beta$-catenin signaling to facilitate regeneration. Therefore, we utilized a wholebody irradiation-induced (WBI-induced) regeneration model to further define the role of SETD2 in the regulation of Wnt signaling. $H \& E$ staining of intestinal paraffin sections was employed to assess the number and length of villus during the regeneration process. Setd $2^{\triangle I E C}$ mice exhibited an accelerated regeneration process in comparison with control littermates. By day 5 after irradiation, intestinal regeneration as measured by villus length was largely recovered in the intestine of Setd $2^{\triangle \mathrm{IEC}}$ mice, but not in control litters (Figure 5, A and B). In accordance with the results in $\mathrm{Apc}^{\mathrm{min} /+}$ mice, Setd 2 deletion significantly enhanced the levels of Wnt/ $\beta$-catenin targets such as Fzd6, Fzd7, Axin2, and Ccnd1 along with increasing expression of the progenitor cell-associated genes Cd133 and Cd44 (Figure 5C). Collectively, our results indicated that SETD2 restricted stem/progenitor cell expansion during intestine regeneration.

To complement our genetic findings, we utilized intestinal organoid culture (also known as mini-guts or enteroids) to directly interrogate the cell-intrinsic response to SETD2 reduction without potential interference by tissue microenvironment. Over 6 days of ex vivo culture, we observed significantly larger sizes of organoids derived from the crypts of $A \mathrm{Ac}^{\mathrm{min} /+}$; Setd $2^{\mathrm{\Delta EC}}$ mice (Figure 5D). In addition, quantitation of organoid differentiation revealed that SETD2 deficiency impaired the sprouting of organoid, a result that is characteristic of a less differentiated phenotype and more reminiscent of tumorigenesis (Figure 5D). In accordance with the faster kinetic growth of SETD2-depleted organoids, phospho-H3 staining indicated that $\operatorname{Setd} 2$ ablation renders the cells more proliferative (Figure 5E). In addition, whole-mount staining revealed the enhanced nuclear translocations of $\beta$-catenin in the absence of SETD2 (Figure 5E). Likewise, SETD2-deficient organoids exhibited mislocalized and increased Paneth cells likely caused by enhanced Wnt signaling (Figure 5E). Further RT-qPCR analysis indicated that these cyst-like structures produced high levels of stem or progenitor cell-associated genes, including Lgr5, Axin2, Olfm4, and Cd133, whereas the terminal differentiation-related genes Alpi, Fabp2, Muc2, and Clca3 were simultaneously downregulated in the absence of SETD2 (Figure 5F). Collectively, these results demonstrate that SETD2 regulates intestinal self-renewal and differentiation in a cell-intrinsic manner.

SETD2 modulates AS of the genes implicated in oncogenesis. Accumulating evidence suggests that SETD2 regulates RNA splicing (10-14). This may reflect the ability of SETD2 to influence transcription elongation and initiation $(5,9)$. Regardless of the molecular mechanisms, loss of SETD2 has been shown to modulate AS in multiple biological contexts. As such, we conducted the Multivariate Analysis of Transcript Splicing (rMATS) program (27) to identify SETD2-regulated AS events during intestinal tumorigenesis. As summarized in Table 1, 711 genes exhibited the signifi- 

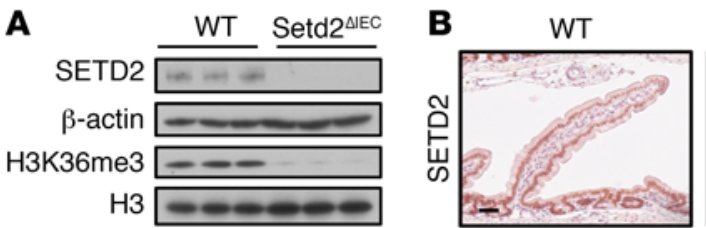

Setd2 $2^{\Delta I E C}$
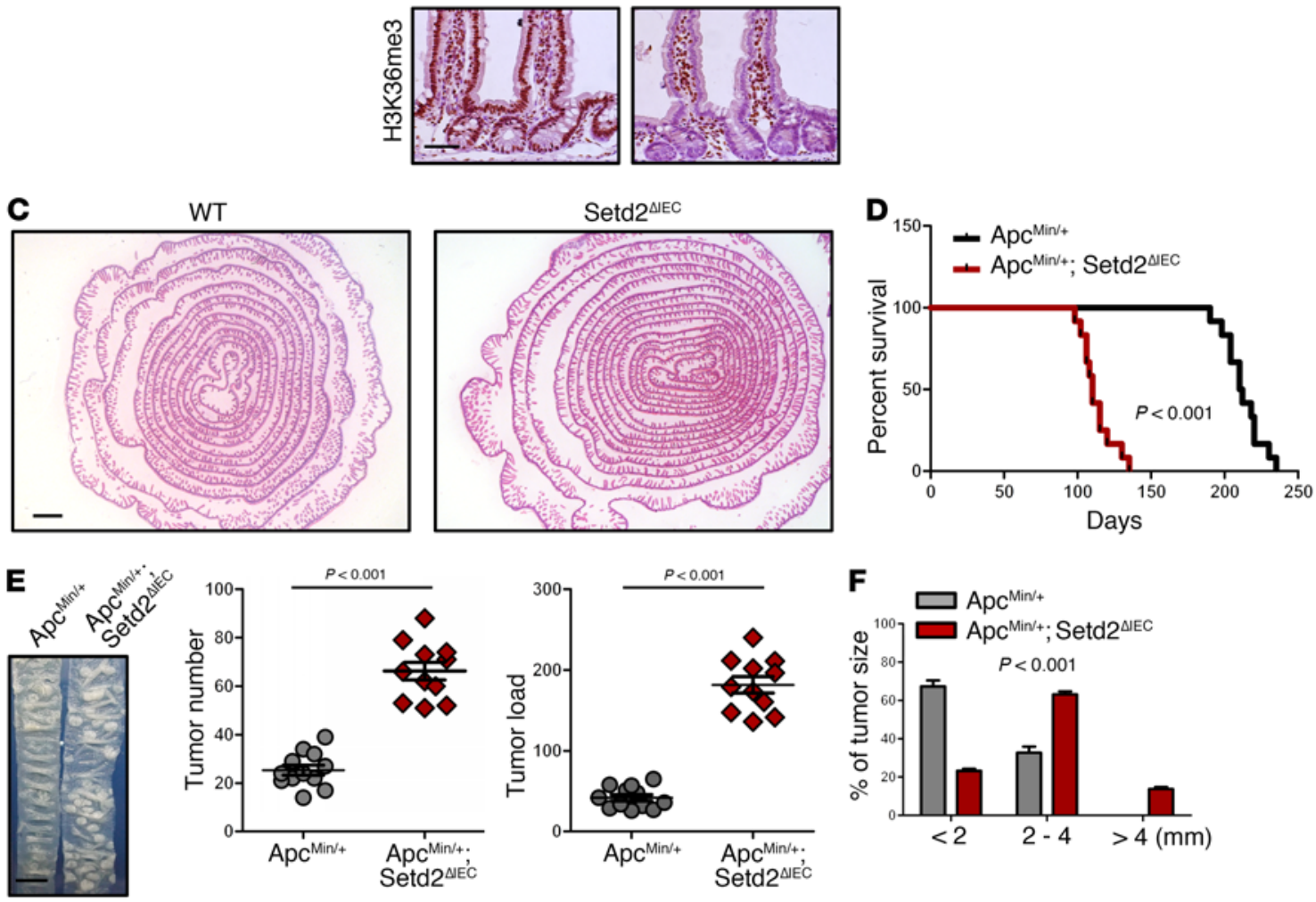

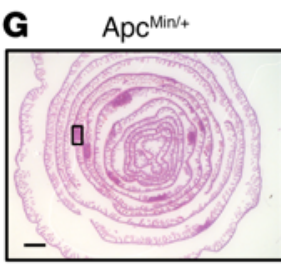

$\mathrm{Apc}^{\mathrm{Min} /+} ;$ Setd2 $^{\mathrm{AEC}}$



$H \& E$

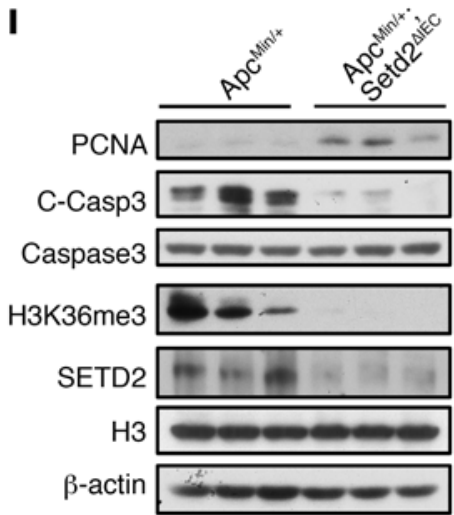

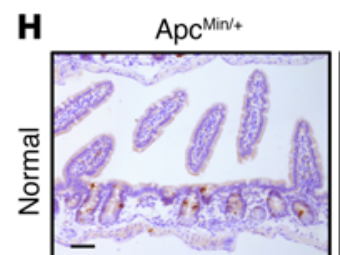


Phospho-H3

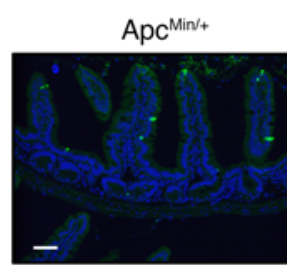

$\mathrm{Apc}^{\mathrm{Min} /+} ; \operatorname{Setd}^{\mathrm{AEC}}$
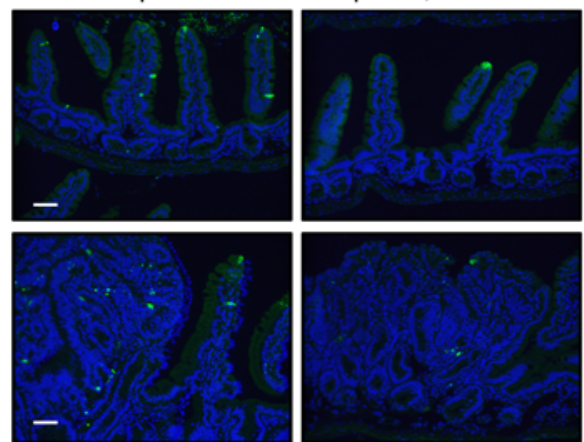

C-Casp3 DAPI
Figure 3. Setd2 inactivation potentiates tumor malignance in an $\mathrm{Apc}^{\mathrm{min} /+}$ mice model. (A and B) Western blot (A) and immunohistochemical staining (B) of the indicated proteins in small intestines of WT and Setd $2^{\Delta \mathrm{EEC}}$ mice. (C) H\&E staining of representative Swiss roll of small intestines of 10-month-old mice. (D) Kaplan-Meier survival plots of $\mathrm{Apc}^{\mathrm{min} /+}$ and $\mathrm{Apc}^{\mathrm{min} /+}$; Setd2 ${ }^{\Delta I E C}$ mice as indicated $(n=12)$ (logrank test). (E) Macroscopic image, tumor number, and tumor load from the small intestines of 14-week-old mice as indicated ( $n=12$ ); 2-tailed Student's $t$ test. (F)

Histogram showing the size distribution of tumors from 14-week-old mice as indicated ( $n=11 ; \chi^{2}$ test). ( $\mathbf{G}$ and $\mathbf{H}$ ) H\&E images (C) and phospho-H3, cleaved caspase 3 staining $(\mathbf{H})$ of the representative small intestines from 14 -week-old $\mathrm{Apc}^{\mathrm{min} /+}$ and $\mathrm{Apc}^{\min /+} ;$ Setd2 ${ }^{\triangle 1 E C}$ mice. Arrowhead denotes the discontinuous muscle layer due to the invasion of tumor cells. (I) Western blot of the indicated protein from small intestine lysates. Blot images are derived from replicate samples run on parallel gels. Scale bars: $50 \mu \mathrm{m}$ (B, G, H, lower panel); $1 \mathrm{~mm}$ (C, G, upper panels); $1 \mathrm{~cm}$ (E). 
A
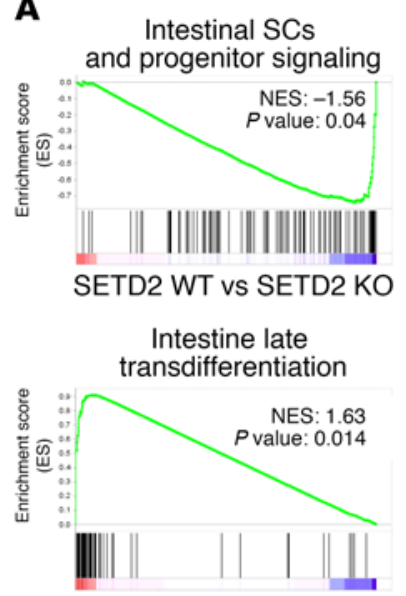

SETD2 WT vs SETD2 KO

C

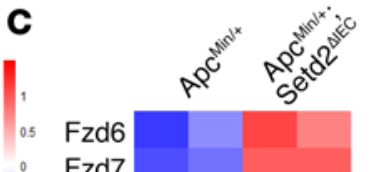

- Fzd7

.05 Lrp5

Axin2

Apcdd1

Myc

Cond1

Ki67

Cd133

Cd44

Ephb2

Ephb3

Sox 9

Ascl2

Olfm4

Lgr5

Setd2

F
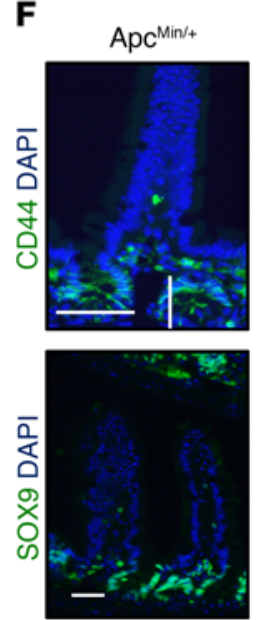

Pece mammary stem cell up signaling

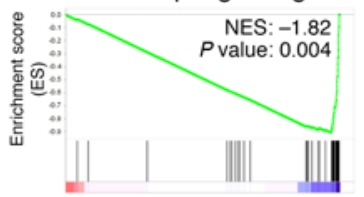

Wnt positive regulated gene in crypts
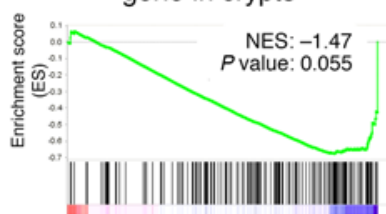

SETD2 WT vs SETD2 KO
SETD2 WT vs SETD2 KO

B


D
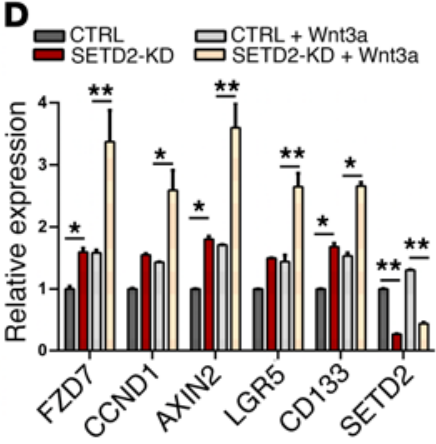

E

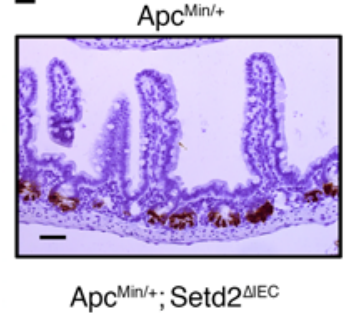

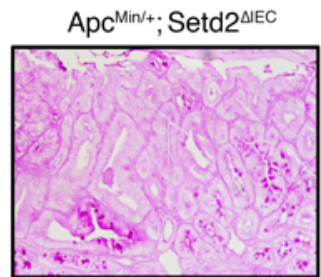
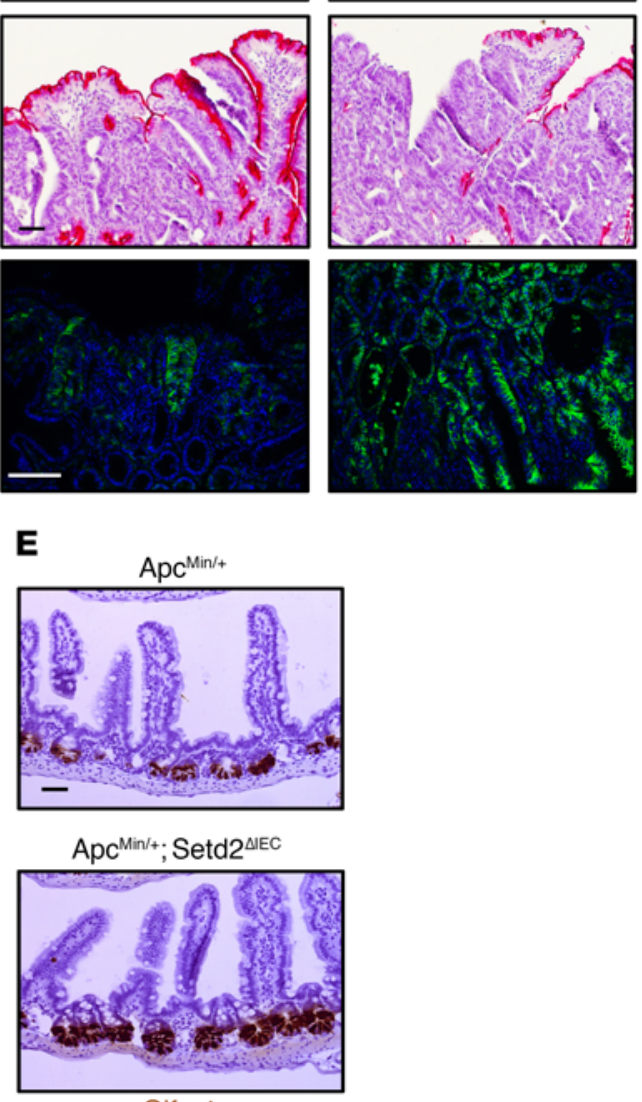

Olfm4

G

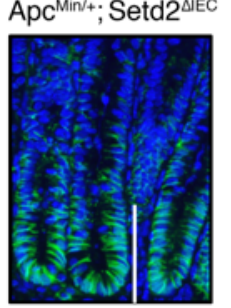

G $\quad \mathrm{Apc}^{\mathrm{Min} /+}$

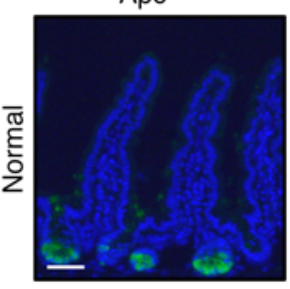

$\operatorname{Apc}^{\mathrm{Min} / 4} ; \operatorname{Setd}^{\mathrm{MIEC}}$

H

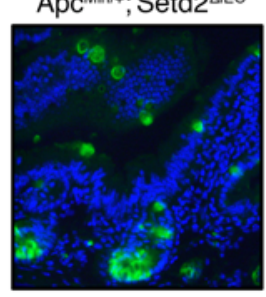

H $\quad \mathrm{Apc}^{\mathrm{Min} /+}$


Lysosome DAPI


$\beta$-catenin

Figure 4. SETD2 loss stimulates Wnt-induced transformation and stemness programs. (A) GSEA enrichment plots of differentially expressed genes belonging to stem/progenitor, differentiation, and Wnt signaling associated with SETD2 downregulation. (B) PAS, ALP, and CD44 staining in intestinal tumors from 14-week-old $\mathrm{Apc}^{\mathrm{min} /+}$ and $\mathrm{Apc}^{\mathrm{min} /+}$; Setd2 ${ }^{\mathrm{AIEC}}$ mice. (C) Heat map summarizing the RT-qPCR results comparing the expression of Wnt target genes in the intestine from 14-week-old mice as indicated. (D) RT-qPCR analysis of Wnt target genes in control and SETD2-knockdown HCT116 cells treated with Wnt3a. Statistical comparisons were made using a 2-tailed Student's $t$ test. (E and $\mathbf{F}$ ) In situ hybridization of stem cells with $\mathrm{Olfm}^{+}(\mathbf{E})$ and immunostaining of the progenitor cells (CD44 and SOX9 positive) (F) from intestine sections as indicated. (G and $\mathbf{H})$ Staining of lysosome and $\beta$-catenin in the adjacent normal and tumor parts of intestine sections as indicated. Scale bars: $50 \mu \mathrm{m}$. ${ }^{*} P<0.05 ;{ }^{* *} P<0.01$. 
A


Figure 5. SETD2 depletion promotes intestinal regeneration and self-renewal. (A) H\&E staining of small intestina sections 3 and 5 days after WBI (10 Gy). (B) The lengths of villus were quantitated in at least 6 fields of small intestinal sections from WT and Setd2 ${ }^{\triangle E E C}$ mice $(n=9)$. (C) Cene expression analysis of small intestine during regeneration (days 0 and 3 ), as indicated. (D) Organoid morphology (left) and quantification of size or differentiation after 6 days of culture (right). Size differences were calculated by Student's $t$ test, and differentiation was measured by sprouting per organoid (Fisher's exact test, $P<0.001$ ). (E and F) Phospho-H3, $\beta$-catenin, and lysozyme whole-mount staining $(\mathbf{E})$ and gene expression analysis $(\mathbf{F})$ of organoid derived from $\mathrm{Apc}^{\mathrm{min} /+}$ and $\mathrm{Apc} \mathrm{cin}^{\mathrm{mi} /}$; Setd $2^{\Delta \mathrm{EEC}}$ mice $(n>4)$. Statistical comparisons in $\mathbf{B}, \mathbf{C}$, and $\mathbf{F}$ were made using a 2-tailed Student's $\mathbf{t}$ test. ${ }^{*} P<0.05$; ${ }^{* *} P<0.01$. Scale bars: $50 \mu \mathrm{m}$ (A); $400 \mu \mathrm{m}$ (D and E).
B

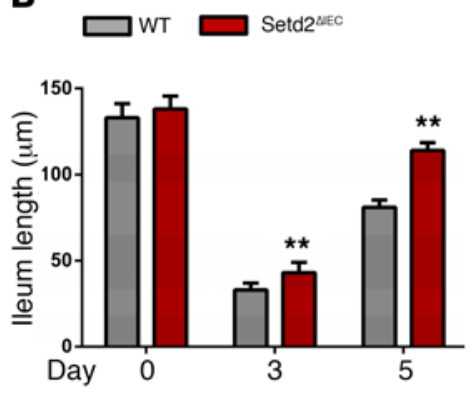

C Fzd6
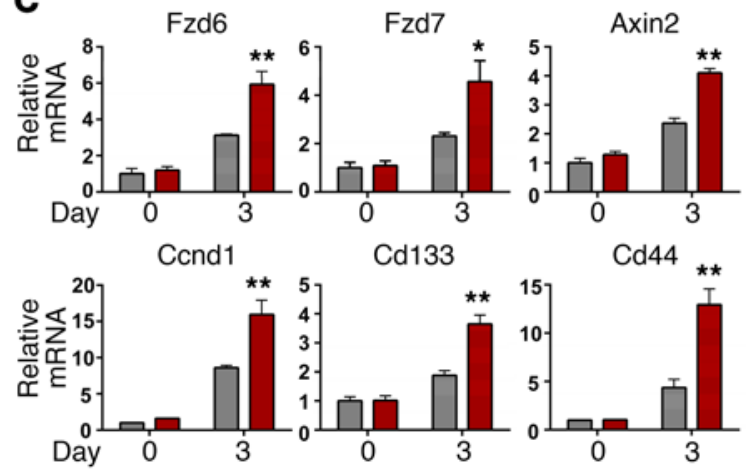

D
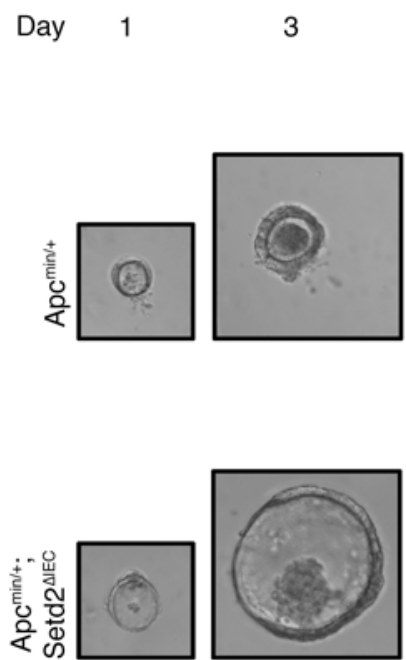

$\mathbf{F}$



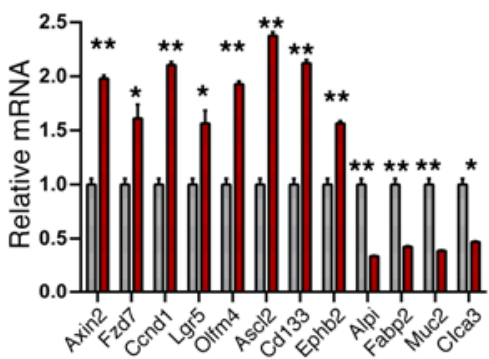

6



Setd ${ }^{N E C}$
E
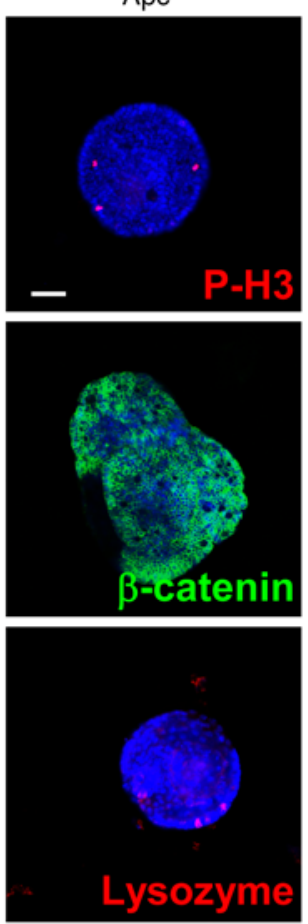

$\mathrm{Apc}^{\mathrm{Min} /} ; \operatorname{Setd}^{\mathrm{NEC}}$
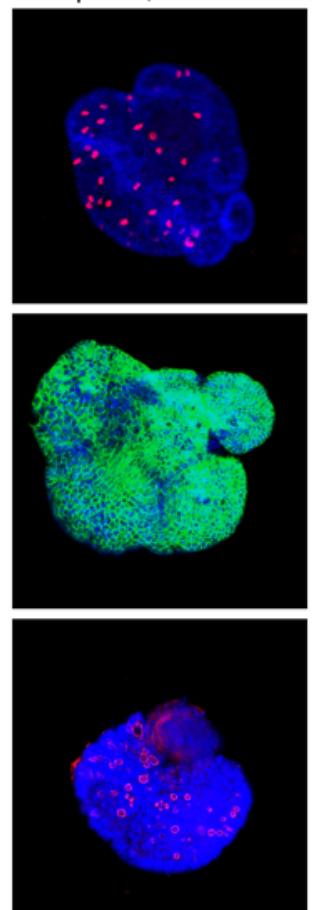
cant alterations of AS in SETD2-deficient intestine versus controls. Interestingly, many of the affected genes were part of recognized cancer-associated pathways (e.g., Sirt7, Cdk4, Cdk7, Rab1a, and Lkb1) or were key signaling components (e.g., Dvl2, Smurf2, Ephb3, and Jag1). Specifically, there were 279 genes with intron retention (IR) (39.2\%) AS events, 198 with exon skipping (SE) (27.8\%), and 161 with mutually exclusive exons (MXE) (22.8\%). Interestingly, 238 out of 279 STED2-influenced IR genes (81.8\%), displayed the reduced IR in the absence of SETD2 (Figure 6A). Likewise, 136 out of 198 STED2-influenced SE genes (68\%) exhibited an increase of exon inclusion, whereas MXE showed no preferences for exon utilization (Figure 6A). These results implied that loss of SETD2 or H3K36me3 within the chromatin facilitates the production of intron-loss or exon-inclusion transcripts in mouse intestine.

To further understand the underlying mechanisms, we conducted ChIP sequencing (ChIP-Seq) to analyze the genomic distributions of $\mathrm{H} 3 \mathrm{~K} 36 \mathrm{me} 3$ in control and SETD2-deficient compound mice. When comparing ChIP-Seq intervals in control and SETD2-deficient intestine, 6,899 genes displayed more than 2-fold reductions of H3K36me3 modifications upon Setd2 ablation, and the altered density of H3K36me3 intervals (Setd2 KO versus control) were mainly localized within gene bodies (Supplemental Figure 3C). Next, we correlated the AS events with SETD2 genomic occupancies and identified that there were a total of 368 out of 771 (51.8\%) genes exhibiting dysregulated AS and the reductions of H3K36me3 modifications after SETD2 depletion. Of note, the overlapping genes were more likely to exhibit the reduction of $\mathrm{H} 3 \mathrm{~K} 36 \mathrm{me} 3$ within the gene body and not within promoters and/or intergenic regions (Figure 6B), highlighting the mechanistic link between lower intragenic H3K36me3 levels and AS. Of note, we found no association between intron length and IR ratio in the presence or absence of SETD2 (Supplemental Figure 3D). The H3K36me3 codes for the representative overlapping genes are presented in Figure $6 \mathrm{C}$ (taken from the ChIP-seq data).

As IR, SE, and MXE represented the most AS changes influenced by SETD2, we further validated the sequencing results by RT-qPCR assays. Results showed less intron inclusions for Ephb3, Sirt7, Jag1, and Cdk4 genes in SETD2-deficient intestinal epithelial cells (IECs) as compared with controls, whereas $\beta$-actin served as the control, displaying no appreciable differences (Figure 6D). Overall, we detected significant differences of IR in all cases (8 genes examined). Intron-retaining transcripts often contain premature stop codons to elicit NMD, resulting in decreased levels of mRNA (28). In line with this notion, the heat map summarizes the IR-related genes and shows their expressions were largely upregulated in SETD-deficient IECs (Supplemental Figure 4A). Similarly, RT-qPCR analysis of preselected genes showed that EphB3, Sirt7, Jag1, and Cdk4 mRNA were increased in the intestinal epithelium lacking Setd2 (Figure 6E). To demonstrate the involvement of SETD2 and H3K36me3 histone marks in this molecular event, we performed ChIP-qPCR analyses to compare H3K36me3 occupancies in the IR locus of Ephb3, Sirt7, Jag1, and Cdk4 genes. Indeed, SETD2 loss eliminated H3K36me3 modifications in the IR regions (Figure 6F), supporting a direct involvement of SETD2. Though MRG15 has been shown to modulate AS by recognizing H3K36me3, we found that depletion of MRG15 in HCT116 cells did not affect the IR events of genes influenced by SETD2 (e.g., DVL2, JAG1, and SIRT7) (Supplemental Figure 4B), suggesting that it is unlikely MRG15 is involved in SETD2-mediated IR. Interestingly, ChIP analysis using antibodies specific for Pol II and Ser2phosphorylated Pol II revealed that SETD2 depletion led to an enrichment of elongated Pol II at the intron-retained locus of Ephb3, Jag1, Cdk4, and Cdk16, but not Sirt7 (Figure 6G). Among a total of 8 genes examined, there were 5 genes displaying the pause of the Pol II elongation rate within the intron-retained locus. These results suggested that slower transcription elongation upon SETD2 depletion might facilitate the removal of intron. Such a kinetic model has been shown as one of the mechanisms for regulating cotranscriptional splicing (11, 29-31). A similar analysis was conducted to examine SE (Eif5a, Ehmt2, and Smurf2) and MXE (Pkm2, Tpm1, and Epn1), and we showed that Setd2 ablation affected SE and MXE types of AS as well (Supplemental Figure 4, C and D). Together, our results indicate that SETD2 regulates RNA splicing, which might contribute to intestinal tumorigenesis.

SETD2 loss augments Wht signaling through regulating NMDcoupled AS of DVL2. Having demonstrated that SETD2 modulated AS, we asked whether these affected genes could explain enhanced Wnt signaling in the absence of SETD2. As such, classical T cell factor (TCF) reporter assay was performed in HCT116 cells. As shown in Supplemental Figure 5A, the level of $\beta$-catenindriven transcription activity, measured by the TOP/FOP luciferase reporter ratio, was compromised in SETD2-overexpressing HCT116 cells. Conversely, depletion of SETD2 enhanced luciferase activity in response to Wnt3a stimulation. Interestingly, we noticed that $\beta$-catenin protein and nonphosphorylated $\beta$-catenin (active $\beta$-catenin) were significantly upregulated in SETD2-silencing cells without any change in mRNA (Figure 7A). Consistent with the enhanced nuclear accumulation of $\beta$-catenin (Supplemental Figure $5, \mathrm{~B}$ and $\mathrm{C}$ ), reduced polyubiquitination of $\beta$-catenin was observed in HCT116 cells depleted with SETD2 (Figure 7B). Collectively, the above results prompted us to investigate whether the protein stability was governed by SETD2. Time-course experiments revealed that depletion of SETD2 in cells augmented $\beta$-catenin stability, whereas MG132 treatment attenuated the destruction of $\beta$-catenin protein (Supplemental Figure 5D), indicating SETD2 regulates $\beta$-catenin stability through $26 \mathrm{~S}$ proteasome. To further substantiate this notion, we treated the cells with lithium chloride ( $\mathrm{LiCl}$ ) to inhibit the destruction complex of $\beta$-catenin and observed that Wnt signaling in control and SETD2-silencing cells was comparable (Supplemental Figure 5E), suggesting that SETD2 modulates Wnt signaling through regulation of $\beta$-catenin stability.

Focusing on the potential candidates implicated in Wnt signaling, we noticed that $D v l 2$ was listed as the top candidate, showing the reduced IR of its intron 2 and displaying significantly fewer H3K36me3 modifications upon Setd2 deletion. DVL-mediated coclustering of FRZ, LRP6, AXIN, and associated kinases at the plasma membrane triggers phosphorylation of the LRP6, thereby promoting $\beta$-catenin stabilization. In addition, DVL also plays a pivotal role in the nucleus by mediating the formation of the $\beta$-catenin/TCF transcriptional complex in conjunction with c-Jun, which is required for Wnt/ $\beta$-catenin signaling $(26,32-34)$. Given the importance of DVL in the regulation of Wnt signaling, we performed RT-PCR analysis and confirmed that Dvl2 pre-mRNA without 
A

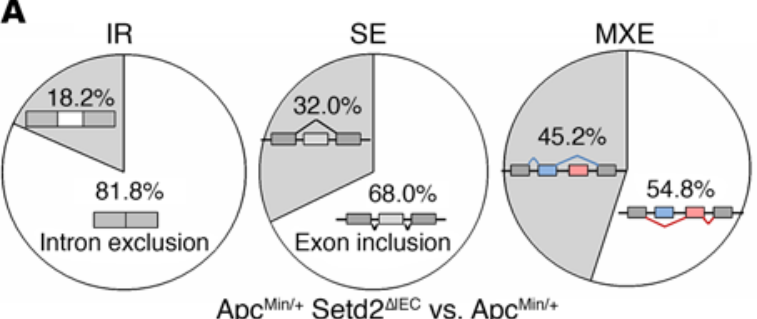

C
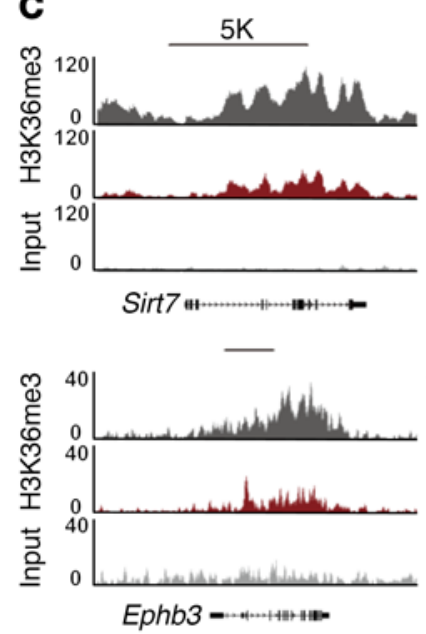

E



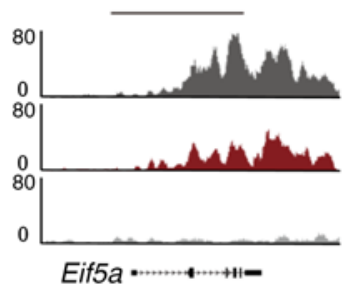

B

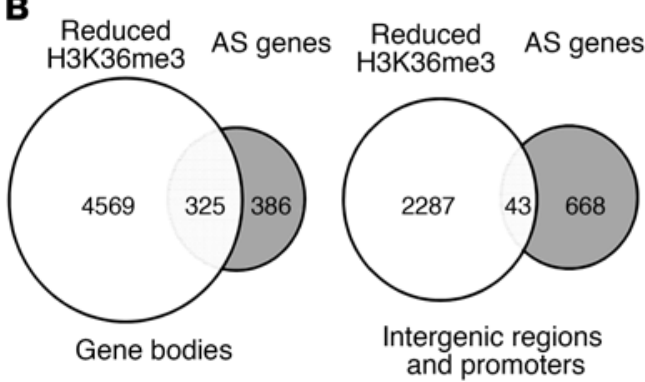

D
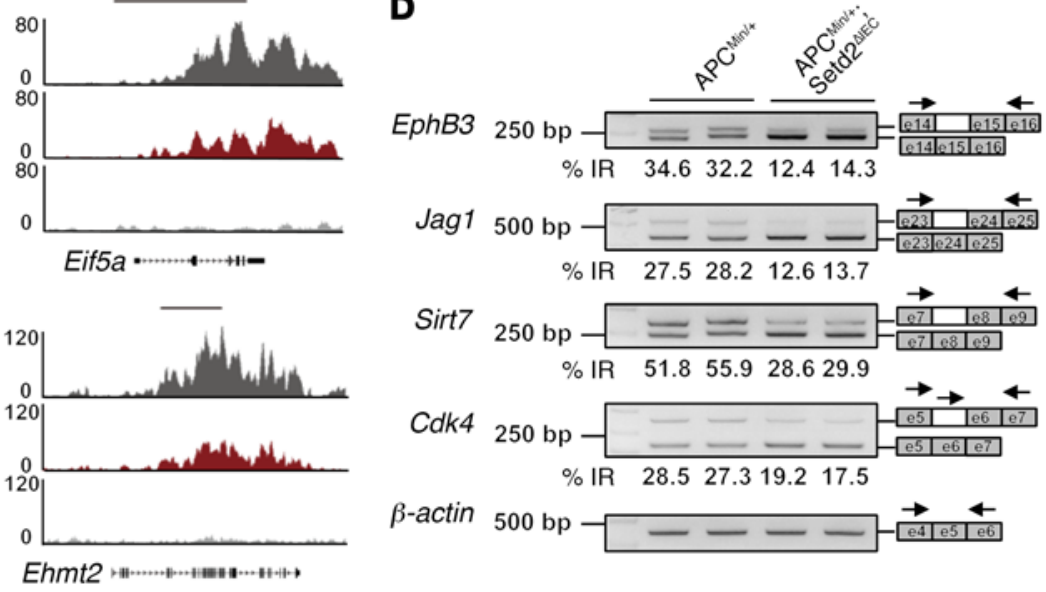

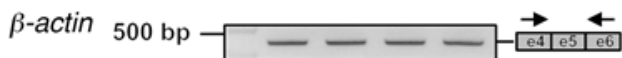

G

Figure 6. SETD2 regulates cancer-associated AS in Apc ${ }^{\min /+}$ mice. (A) Pie graph summary of AS in SETD2 KO mice versus control mice. (B) Venn diagram indicates that altered AS genes (gray) preferably exhibited the decrease of $\mathrm{H} 3 \mathrm{~K} 36 \mathrm{me} 3$ codes within gene bodies in comparison with the promoter and intergenic regions ( $P<0.001 ; \chi^{2}$ test). (C) Snapshot of H3K36me3 ChIP-Seq signal at the represented gene locus in control and Setd2-knockout mice. (D and E) Knockout of Setd2 in intestinal epithelium causes a decrease of IR (D) and an increase of mRNA levels of the indicated genes (E). Primers for examinations of IR are indicated by the arrows. (F) H3K36me 3 codes in the areas of IR-related genes in the IECs of $\mathrm{Apc}^{\mathrm{min} /+}$ and $\mathrm{Apc}^{\mathrm{min} /+}$; Setd2 $2^{\Delta \mathrm{IEC}}$ mice. (C) ChIP-qPCR analysis of total Pol II and Ser2-phosphorylated Pol II enrichments in the IR gene locus, as indicated by number in control and SETD2-deficient Apc-mutated IECs. Statistical comparisons in E-G were made using a 2-tailed Student's $t$ test. ${ }^{*} P<0.05 ;{ }^{* *} P<0.01$.

intron 2 was profoundly increased in SETD2-deficient intestines (Figure 7C). ChIP-qPCR analysis verified that Setd 2 ablation diminished H3K36me3 modifications within the IR areas of the Dvl2 gene locus (Supplemental Figure 6A), indicating a direct involvement of SETD2 in regulating the processing of DVL2 pre-mRNA. In agreement with the results shown in Figure 6G, ChIP-qPCR analysis using antibodies specific for Pol II-phosphorylated CTD at Ser2, an elongating form of Pol II as well as total Pol II, revealed that SETD2 depletion led to paused Pol II at the intron-retained locus of Dvl2 (Figure 7D). These results suggested that slower transcription elongation upon SETD2 depletion might facilitate the removal of intron 2.

We noticed that the intron 2-containing DVL2 transcript carries a premature stop codon in intron 2, suggesting that this transcript is potentially subjected to NMD regulation. To confirm SETD2mediated DVL2 expression through NMD, we used siRNA to knock down UPF1, which is a central factor in NMD. Depletion of UPF1 in HCT116 cells led to the increase of intron-retained transcripts of $D V L 2$ (Figure 7E), indicating that the DVL2 transcript with intron 2 is indeed subjected to NMD. In line with this notion, Dvl2 mRNA was profoundly increased in SETD2-deficient intestines (Supplemental Figure 6B). Similarly, SETD2 depletion increased DVL2 expression in CRC cells, whereas overexpression of SETD2 in HCT116 cells reduced its expression (Supplemental Figure 6, C and D). We also detected the enhanced or reduced nuclear accumulation of DVL2 in SETD2-depleted and -overexpressed cells (Supplemental Figure 6, $\mathrm{E}$ and $\mathrm{F}$ ). Of note, we did not see the decrease of nuclear $\beta$-catenin in SETD2-overexpressed cells (Supplemental Figure 6F), which is presumably due to the compensatory role of DVLs in cell cultures. Together, our data reveal that SETD2 depletion increases DVL2 mRNA expression through NMD-coupled AS regulation.

We hypothesized that the upregulation of DVL2 in the absence of SETD2 led to the increase of both stabilization and transcription activity of $\beta$-catenin and thereby augmented Wnt signaling. To clarify this issue, we depleted DVL2 expression in SETD2knockdown cells. As reflected by the expression of Wnt targets, reduced DVL2 expression in SETD2-silencing cells diminished Wnt activity and oncosphere formation to levels similar to those of control cells (Supplemental Figure 6, G and H). More importantly, to directly assess the functional dependence between DVL2 IR and SETD2 effects in tumorigenesis, we utilized antisense oligonucleotide (splicing switching oligonucleotide [SSO]) (35) to induce intron 2 retention of DVL2 in SETD2-deficient cells (Figure $7 \mathrm{~F}$ ). As indicated by the number of oncospheres, we showed that enhancement of IR for DVL2 largely diminished the growth advantage elicited by SETD2 loss (Figure 7G). These results suggested that SETD2 exerts its tumor-suppressive role largely dependent on the regulation of DVL2 splicing. Similar results were obtained in GEMs, that is, SETD2 loss led to increased DVL2 expression and thus upregulated Wnt activity, as evidenced by the enhanced phospho-LRP6 levels and nuclear accumulation of DVL2 and $\beta$-catenin from Apc ${ }^{\min /+} ;$ Setd2 ${ }^{\triangle \mathrm{IEC}}$ mice (Figure 7, H and I). The clinical relevance of DVL2 and SETD2 was substantiated by patient specimen analysis. We showed that DVL2 mRNA levels were negatively correlated with SETD2 transcripts in colorectal tumors (Supplemental Figure 6I). Collectively, our results indicate that SETD2 loss induces DVL2 mRNA expression and thereby leads to hyperactivation of Wnt signaling.

SETD2 loss aggravates Wnt/ $\beta$-catenin-dependent CRC progression. To clarify whether SETD2 loss-mediated tumorigenesis is dependent on hyperactivation of Wnt signaling, we silenced $\beta$-catenin in HCT116 cells and found that depletion of $\beta$-catenin in SETD2-deficient cells compromised oncosphere growth to an extent similar to that seen with control cells (Supplemental Figure 7A). Conversely, knockdown SETD2 in constitutively active $\beta$-catenin overexpression cells did not further enhance anchorageindependent growth as compared with $\beta$-catenin overexpression cells (Supplemental Figure 7B). Together, these results highlighted that SETD2 exerts its tumor-suppressive function in a manner largely dependent on Wnt signaling. To further substantiate this notion, we performed an intrasplenic injection of metastatic assay to clarify the functional relevance of SETD2 and Wnt signaling in CRC. To achieve this, HCT116 cells stably expressing luciferase were injected and spleen was resected 2 minutes after injection to avoid the primary lesion effect. As measured by bioluminescent imaging, overexpression of constitutively active $\beta$-catenin or depletion of SETD2 enhanced the distal colorizations of colorectal tumor cells within the liver 1 month after inoculation (Figure 8, A and B). More importantly, depletion of SETD2 did not further enhance metastasis induced by $\beta$-catenin overexpression alone (Figure $8, \mathrm{~A}$ and B). Similarly, the bioluminescent imaging results could be confirmed by the macroscopic image and $\mathrm{H} \& \mathrm{E}$ staining of metastatic lesions in liver (Figure 8C and Supplemental Figure 7C). Finally, the functional relevance between SETD2 and $\beta$-catenin signaling could be illustrated by transcriptomic signature analysis in patients. There was a significant and negative correlation among SETD2, $\beta$-catenin, LGR5, and ISCs and progenitor signature in colorectal tumors (using data sets from NCBI's Gene Expression Omnibus [GEO] GSE35982 and GSE17538; Figure 8D). Collectively, our results highlight that SETD2 promotes CRC progression in a manner largely dependent on Wnt/ $\beta$-catenin signaling.

\section{Discussion}

Intestinal tumorigenesis is thought to originate from ISCs and requires multiple subsequent mutations in the genes implicated in cell growth, differentiation, and survival. ISCs require the Wnt signal for its self-renewal and differentiation; however, an uncontrolled Wnt pathway leads to the expansion of stem or progenitor cells and aberrant lineage decisions $(23,36)$. Several studies have linked H3K36 methylation to abnormal differentiation or proliferation. As 
A

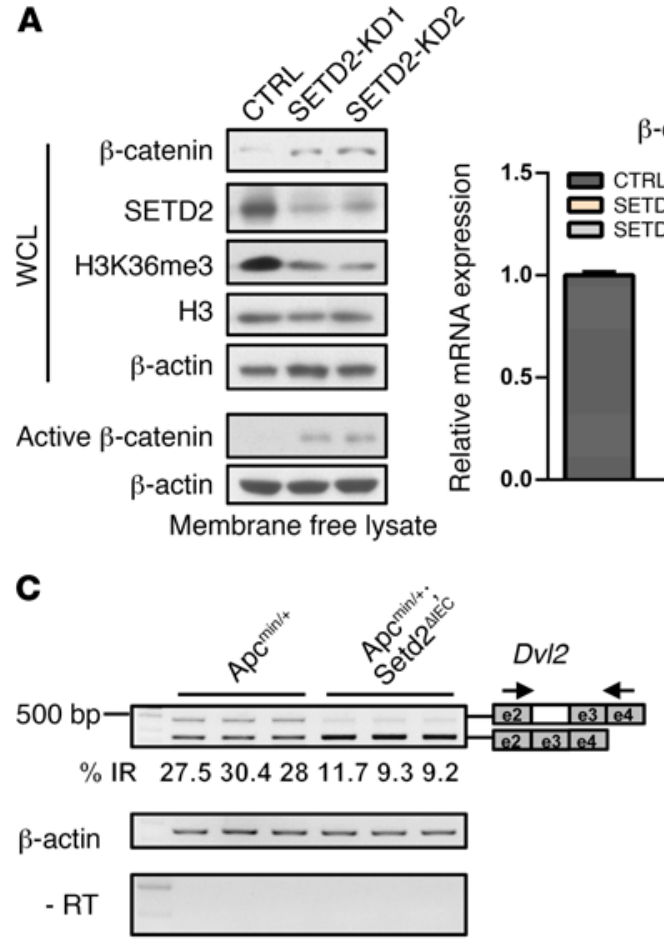

B

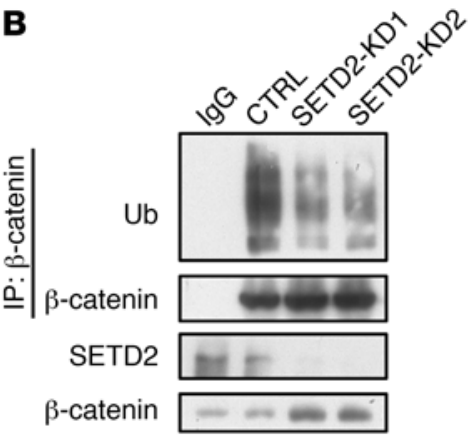

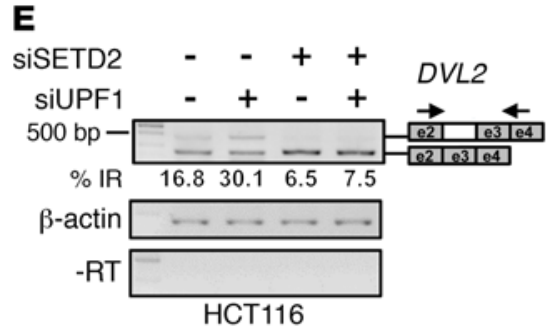

F

D $\square A p c^{M i n / *}$
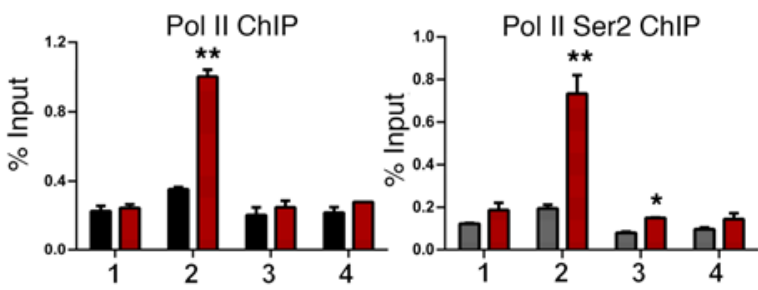

G


SETD2-KD SETD2-KD + SSO


Figure 7. SETD2 counteracts Wnt/ $\beta$-catenin pathway through regulation of NMD-coupled splicing of DVL2 pre-mRNA. (A) Western blotting analysis (left) and RT-qPCR analysis (right) of the indicated genes in control and SETD2-knockdown HCT116 cells. Cytomembrane-free lysate was used to examine active $\beta$-catenin. (B) Western blotting analysis of the indicated protein and immunoprecipitates derived from HCT116 cells. Blot images are derived from replicate samples run on parallel gels. (C) RT-PCR analysis of Dvl2 splicing patterns in IECs as indicated. Upper band intron-retained transcripts. The average percentages of IR are shown. (D) ChIP-qPCR analysis of Pol II and Ser2-phosphorylated Pol II enrichments in the Dvl2 gene locus, as indicated by number in control and SETD2-deficient Apc-mutated IECs.

(E) RT-PCR analysis of DVL2 splicing patterns in control and SETD2-knockdown HCT116 cells with or without UPF1 knockdown. ( $\mathbf{F}$ and $\mathbf{G}$ ) Control and SETD2-knockdown HCT116 cells transfected with control or SSO. SSO oligonucleotide target against 3 ' splicing site of intron 2 of DVL2. (F) RT-PCR analysis of DVL2 mRNA and the average percentages of IR are shown. (G) Oncosphere formation of the cells as indicated. Quantitation results are shown in the right panel. ${ }^{*} P<0.05$; ${ }^{* *} P<0.01$. (H) Western blot analysis of the small intestine lysates from $\mathrm{Apc}^{\mathrm{min} /+}$ and $\mathrm{Apc}^{\mathrm{min} /+}$; Setd2 $2^{\Delta \mathrm{EEC}}$ mice. (I) Examination of the indicated protein in cytoplasm and nucleus lysates from $\mathrm{Apc}^{\mathrm{min} /+}$ and $\mathrm{Apc}^{\min /+} ;$ Setd $2^{\Delta \mathrm{IEC}}$ mice. Primers used for examination of IR are indicated by arrows. Scale bars: $1 \mathrm{~mm}$. Statistical comparisons in $\mathbf{D}$ and $\mathbf{G}$ were made using a 2-tailed Student's $t$ test 
reported, SETD2 loss impairs ES cell differentiation (37) and expression of H3.3 mutants that inhibit H3K36 methylation compromises the differentiations of chondrocytes and mesenchymal progenitor cells $(38,39)$. The Wnt pathway involves various feedback loops that balance the opposing processes of cell proliferation and differentiation. Studies indicate that, even in the presence of heterozygousactivating mutations downstream of the FRZ receptor, mutationdriven Wnt-signaling activation can be further enhanced in $\mathrm{APC}^{\mathrm{min} /+}$ mice and cells (40-45). Our results highlighted that SETD2 fine-tuned Wnt signaling to safeguard ISCs or progenitor cells in the intestine, whereas downregulation facilitated inherently more proliferative and progenitor traits in ISCs in the Apc-mutated background (Figure 8E). Of note, though our results indicated that SETD2 exerted its function in a manner largely dependent on canonical Wnt signaling, several other key signaling regulators (e.g., JAG1, LKB1, SIRT7, and PKM2) were altered in the absence of SETD2. Thus, other signaling pathways might also contribute to SETD2's impact on intestinal tumorigenesis and regeneration.

Mutation and deletion in the SETD2 gene have been reported in several malignancies; however, the roles of SETD2 loss in cancer development have not been well established. In a very recent study, SETD2 is shown to inhibit lung cancer initiation and progression using a CRISPR-based virus infection approach (46). Here, we took advantage of GEMs and established SETD2 as a negative regulator of Wnt signaling to control excessive stem/progenitor cell proliferation during tissue regeneration and tumorigenesis. Mechanistic studies revealed that SETD2 loss upregulated DVL2 expression despite the mutated destruction complex, emphasizing the importance of SETD2 for controlling Wnt signaling, IEC proliferation, and CRC (Figure 8E). DVL is required for LRP6 phosphorylation $(47,48)$, which is essential for subsequent steps of signal transduction, such as Axin recruitment and cytosolic $\beta$-catenin stabilization. In addition, nuclear DVL cooperates with c-Jun to regulate gene transcription stimulated by the canonical Wnt-signaling pathway $(26,32-34)$. The present study proposes that SETD2 loss leads to upregulated DVL2 expression, which results in the increase of both stabilization and transcription activity of $\beta$-catenin and consequently overrides the threshold of Wnt signaling to facilitate tumor malignance (Figure 8E). Though Dsh homologues (DVL1, -2, and -3) exhibit the redundant roles for regulation of Wnt signaling in the cell culture model, $\mathrm{Dvl}$ knockout mice exhibit the distinct phenotypes $(49,50)$, possibly due to their restricted expressions in vivo. Of note, a previous report determined that DVL2 loss protects against tumor development by reducing Wnt signaling in $\mathrm{Apc}^{\mathrm{min} /+}$; Dvl2 ${ }^{\mathrm{AEC}}$ mice (51), suggesting a nonredundant function of DVL2 for tumorigenesis in $\mathrm{Apc}^{\mathrm{min} /+}$ mice. In addition, we noticed that several Wnt regulators, including WNK2, LKB1, $\beta$-arrestin 2, and RAF1, were altered in the absence of SETD2 $(52-55)$. For instance, $\beta$-arrestin 2 is essential for the initiation and growth of intestinal tumors displaying elevated Wnt pathway activity in Apc-mutated mice (54), and WNK2 also stimulates the Wnt signal through regulation of $\beta$-catenin stability (52). Thus, we cannot completely rule out the possibility that, in addition to our findings, SETD2 might also act on different Wnt pathway components to finetune Wnt signals during intestinal regeneration and tumorigenesis.

SETD2-catalyzed H3K36me3 is associated with transcription elongation, AS, and mRNA export $(5,30,56)$. Similarly to studies in yeast, recent studies in mouse ES cells revealed that
H3K36me3-mediated recruitment of DNMT3b to the gene bodies inhibits spurious transcription initiation (9), implying a possible mechanism for SETD2 loss eliciting abnormal transcripts in cancer cells. As H3K36 trimethylation levels differ based on exon utilization (57-59), several investigations have implicated $\mathrm{H} 3 \mathrm{~K} 36 \mathrm{me} 3$ in alternative splice site choice (10-14). Studies show that altering SETD2 levels influences the inclusion of exons in Fgfr2 genes, indicating that H3K36me3 influences the recruitments of splicing factors (MRG15 and PTB) (14). Likewise, siRNA knockdown of SETD2 in 293T cells results in significant AS alterations (12). In Apc-mutated IECs, we also demonstrated that Setd2 modulated NMD-coupled splicing of Dvl2. Our results indicate that slower transcription elongation upon SETD2 depletion is one of mechanisms for facilitating the removal of intron. However, whether and how this modification directs the changes in splicing remains largely unclear. Chromatin structure and histone modifications have been inferred as affecting AS through 2 models, the kinetic model and the recruitment model (11, 29-31). It is notable that these 2 models are not mutually exclusive, as histone modifications recruit chromatin-modifier enzymes that alter RNAPII elongation, and some histone modifiers or readers associate with RNAPII to affect transcription elongation. We believe that further dissection of SETD2-mediated histone modification and its interaction with specific binding partners (reader) will improve the understanding of how SETD2 influences AS.

Interestingly, our results indicate that IR represents the most altered AS events in Setd2-deficient intestine. These results are consistent with studies showing that histone reader BS69 specifically recognizes that SETD2 catalyzes H3.3K36me3 and its depletion primarily leads to intron exclusion in an $\mathrm{H} 3 \mathrm{~K} 36 \mathrm{me} 3$-dependent manner (13). Since IR has been shown to be a mechanism of tumorsuppressor inactivation (60), it is of importance to further determine how AS is regulated by histone modifications, which will shed insights on the role of SETD2 in the regulation of splicing as well as tumorigenesis. Present studies also indicated that SETD2 loss results in the accumulation of DVL2 mRNA expression through NMDcoupled AS regulation. As indicated by the previous study, about $35 \%$ of AS genes are predicted to be subject to NMD, and this percentage is even higher for IR events (61). As a proof of principle, we used siRNA to knock down UPF1 and showed that depletion of UPF1 in HCT116 cells led to the increase of intron-retained transcripts of SIRT7 and JAG1, but not EPHB3 (Supplemental Figure 8). Thus, DVL2 is not the only gene affected by NMD upon SETD2 depletion.

In summary, our findings highlight the suppressive roles of SETD2 in Wnt signaling, intestinal regeneration, and CRC, with potential implications for disease intervention. In addition, our results establish a role for SETD2- or H3K36me3-mediated AS in intestinal tumorigenesis and may guide the understanding of development and progression of other human disorders associated with SETD2 deficiency.

\section{Methods}

For expression plasmids, shRNA, cell cultures, IEC isolation, immunohistochemistry, organoid culture, RT-qPCR, ChIP-qPCR, and primers (Supplemental Tables 2 and 3), see Supplemental Methods.

Animal experiments. All mice were maintained in a specific pathogen-free facility. SETD2-floxed mice were generated by Shang- 
A

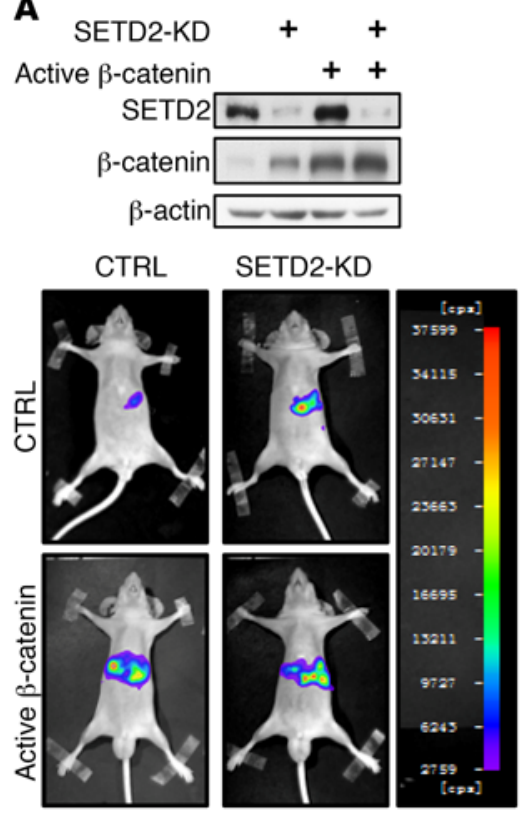

B

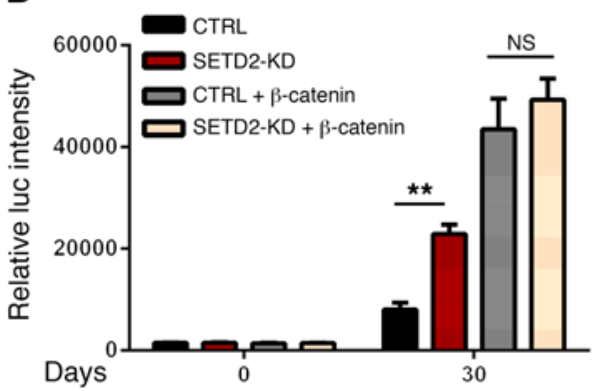

C
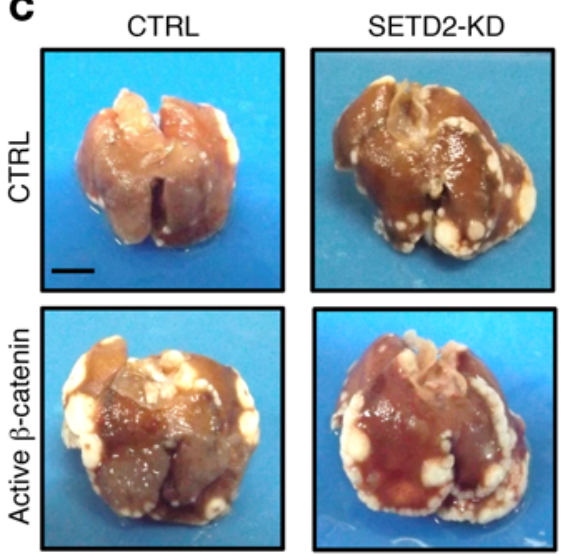

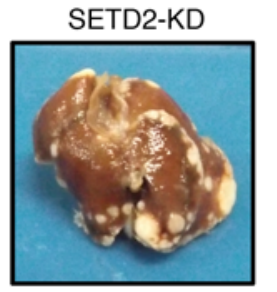

Figure 8. SETD2 loss aggravates Wnt/ $\beta$-catenin-dependent CRC progression. (A) Western blot analysis of the indicated protein (left) and the representative bioluminescence images for tumor metastasis to the liver in control and SETD2-KD HCT116 cells with or without active $\beta$-catenin overexpression (right panel). Blot images are derived from replicate samples run on parallel gels. (B) $B L I$ quantitation of liver metastasis calculated by means \pm SEM of bioluminescent signals is shown $(n=6)$; 2 -tailed Student's $t$ test. ${ }^{*} P<0.01$. (C) Macroscopic images of HCT116 metastasis to liver. (D) Correlations (by Pearson's) between SETD2 signature, $\beta$-catenin signature, LGR5 signature, SCs, and progenitor signature within CRC specimens (GEO GSE35982 and GSE17538) are shown. Yellow, high-signature scoring; blue, low-signature scoring. (E) Model of SETD2 in colorectal tumorigenesis. SETD2 fine-tunes Wnt/ $\beta$-catenin signaling to safeguard intestinal self-review and differentiation largely through modulation of IR and NMD of DVL2 pre-mRNA. Scale bars: $1 \mathrm{~cm}$.

D

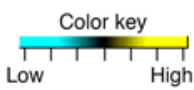

Low 1 T I T

SETD2 signature

$\beta$-catenin signature

LGR5 signature

SCs and progenitor signature
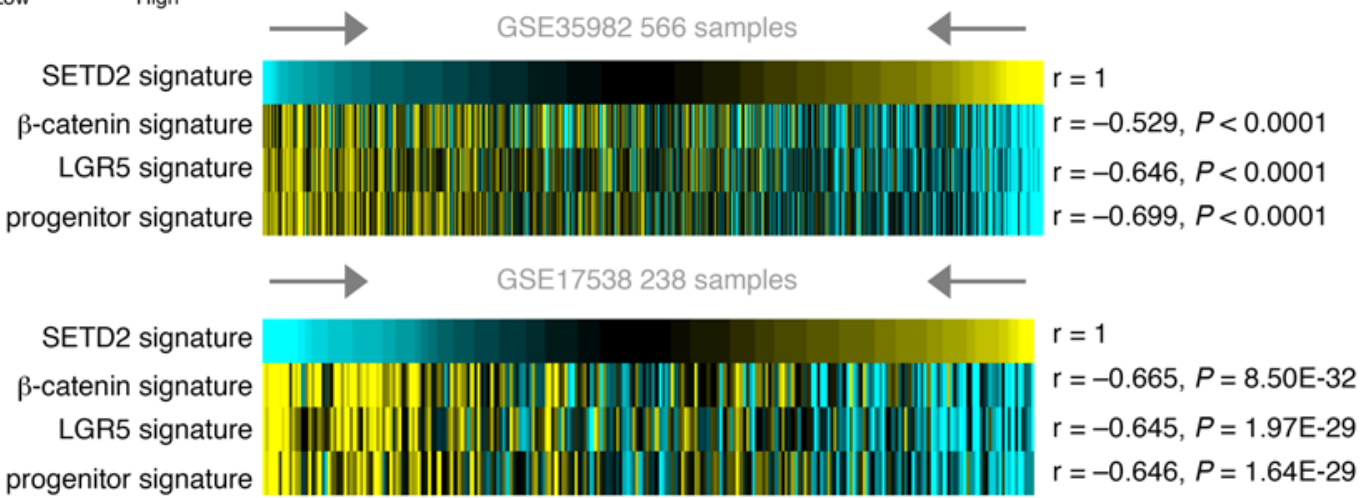

SCs and progenitor signature

\section{E} Normal colon
SETD2/H3K36me3 higher

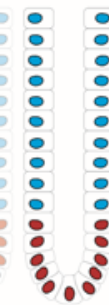

APC mutation

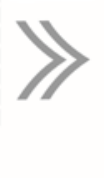

Adenoma

SETD2/H3K36me3 higher



SETD2 loss

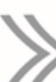


hai Biomodel Organism Co. using conventional homologous recombination in ES cells (provided by Li Li, Shanghai Jiao Tong University, Shanghai, China), and the targeting strategy is indicated in Supplemental Figure 1A. The Apc ${ }^{\text {min }}$ mice and Villin ${ }^{\mathrm{Cre} /+}$ mice were from The Jackson Laboratory. All mice were maintained on a C57BL/6 background. For intraspleen injection, male nude mice were injected with $10^{6}$ HCT116 cells, and tumor formation was monitored by BLI using a NightOWL II LB 983 Imaging System (Berthold).

Human specimen analysis and immunohistochemical analysis of human tissue microarrays. Biospecimens confirmed by 2 independent pathologists were collected from patients with CRC undergoing surgical resection. Tissue RNA or protein was also prepared from the biopsies for analysis of human SETD2 transcript levels in tumor and normal counterparts. Tissue microarrays used in this study included $168 \mathrm{CRC}$ and 48 normal specimens. Briefly, clinical parameters of 168 colon cancer patients were collected, including age, sex, stage, pathological diagnosis, differentiation status, TNM status, and follow-up events (recurrence and metastasis). None of the patients underwent preoperative chemotherapy or radiation therapy prior to surgery. Immunohistochemical analyses were performed as described (62), using anti-SETD2 antibody (generated by Ango Technology). The specificity of this antibody was prevaluated by Setd2-KO mice and SETD2 knockdown cells. Samples were scanned using a Bliss automated slide scanner to generate high-resolution digital images. The expression of protein was scored and quantified by a pathologist (J. Peng) blinded to the outcome of the cases. Basically, the quantified method was based on a multiplicative index of the average staining intensity (from 0 to 3 ) and extent of staining (from 0 to 3 ) in the cores, yielding a 10-point staining index, so that the staining index ranged from 0 (no staining) to 9 (extensive, strong staining).

WBI. Eight-week-old mice underwent 10-Gy WBI using a Shepherd $137 \mathrm{Cs}$-ray irradiator at a dose rate of $4.21 \mathrm{~Gy} /$ minute. The number and length of regenerating villi were quantified ( 24 fields per genotype using $\times 20$ magnification) after $\mathrm{H} \& \mathrm{E}$ staining of paraffin sections (6 animals per group).

RNA-Seq and AS analysis. Intestinal mRNA was obtained from 2-month-old Apc ${ }^{\mathrm{min} /+}$ and $\mathrm{Apc}^{\mathrm{min} /+}$; Setd $2^{\mathrm{AEC}}$ mice, and each RNA sample combined a mixture of RNA from 3 animals. RNA was subjected to Hi-Seq and PE100 (deep sequencing for AS) performed by BGI Tech Solutions Co. Gene expression levels were quantified by the software package RSEM (https://deweylab.github.io/RSEM/). The list of significance was determined by setting a false discovery rate (FDR) threshold at a level of 0.05 and a $P$ value of less than 0.05. All differentially expressed gene lists generated were subsequently analyzed for enrichment of biological themes using the DAVID bioinformatics platform (https://david.ncifcrf.gov/) and the Ingenuity Pathways Analysis program (http://www.ingenuity.com/index.html). For AS analysis, we mapped RNA-Seq reads to mouse transcripts (Ensembl, release 65) and genome (mm9) using TopHat2 (63), allowing 2-bp mismatches per 25-bp seed. rMATS.3.0.9 (27) was used to identify 22,205 regulated differential AS events corresponding to all 5 types of AS patterns. 773 AS genes $(P<0.05, q<0.2)$ were identified with significant difference between Setd 2 knockout and control.

ChIP-Seq assay and data analysis. We conducted ChIP-Seq using the intestines from 2-month-old control $\mathrm{Apc}^{\mathrm{min} /+}$ and $\mathrm{Apc}^{\mathrm{min} /+}$; Setd2 ${ }^{\mathrm{AIEC}}$ mice (each sample contained 4 animals). The chromatin was prepared and followed by ChIP-Seq analysis by Active Motif Inc. using the antibody against H3K36me3 (Active Motif). The 75-nt sequence reads generated by Illumina sequencing were mapped to the genome using the BWA algorithm with default settings. Analysis of SICER data with an FDR cut-off for significance of $1 \times 10^{-10}$ identified 15,037 intervals (control) and 17,847 intervals (SETD2-KO) over input control. Average of peak values of all active regions in the gene and within the gene margin were used to calculate the differentially enriched genes.

Splicing assay. Total RNA was subjected to genome DNA (gDNA) eraser treatment for 5 minutes, followed by reverse transcription using random primers for each sample (Takara, PrimeScript RT Reagent Kit with gDNA Eraser). For IR analyses, cDNA samples were subjected to real-time qPCR analyses with primers designed for the retained introns (In) and the adjacent exons (All); the IR ration (inclusive $\%$ ) was calculated as $\left.100 \% \times 2^{[\mathrm{Ct}(\mathrm{All})}-\mathrm{Ct}(\mathrm{In})\right]$, where Ct represents the cycle value determined by real-time PCR. The PCR products for retained intron were analyzed by gel electrophoresis and quantitated by Kodak Image Station 4000R, the IR ratios were calculated as Intensity $_{\text {(Inclusive) }} /$ Intensity $_{\text {(Inclusive) }}+$ Intensity $_{\text {(Exclusive) }}$. For ES analyses, the same cDNA samples were subjected to real-time qPCR amplification with primers corresponding to the alternatively spliced exons (SE) and adjacent exons (All); the SE ratio was calculated by the following formula: $\%$ Inclusive $=100 \% \times 2^{[\mathrm{Ct}(\mathrm{All})-\mathrm{Ct}(\mathrm{Se})]}$, and the values were normalized to the control groups. A similar assay was used for MXE analyses.

GSEA analysis. The ISCs and progenitor signature that contained 367 significantly highly expressed genes in colon crypt compared with colon top was derived from GEO GSE6894 (64). The Pece Mammary Stem Cell up signature was derived from KEGG c2.all.v5.2. Intestine late transit-amplifying markers were from Grimm et al. (65). The Wntpositive regulated genes in crypt signatures, derived from Fevr et al. (66), contained 557 downregulated genes in intestinal crypt cells upon deletion of $\beta$-catenin. Genes were ranked by "differ of classes," and permutation type was "gene set" and other sets using the GSEA default $(P<0.05$ and $q<0.25)$.

Analysis of SETD2, $\beta$-catenin, LGR5, and SC progenitor signatures in human colon cancer patients. Analysis in human tumor data sets was carried out essentially as previously described (62). The SETD2 gene signature was derived from our own gene expression profile data set. The $\beta$-catenin signature was derived from Fevr et al. (66), and the LGR5 gene signature was from GEO GSE46200, in which normal mouse colons were flow-sorted into Lgr5 high and low cells before gene expression was measured. The SCs and progenitor signatures were derived from GEO GSE6894 (64), which contained 367 significantly highly expressed genes in colon crypt compared with colon top. In order to define the degree of gene signature manifestation within profiles from external human colon tumor data sets (e.g., GEO GSE35982), we used the previously described $t$ score metric $(62,67)$, which was defined for each external profile as the 2-sided $t$ statistic comparing the average of the SETD2-induced genes with the average of the SETD2-repressed genes (genes within the human tumor data set were first centered to SD from the median of the primary tumor specimens). For a given data set, the $t$ score contrasted the patterns of the SETD2-induced genes with those of the SETD2-repressed genes to derive a single value denoting coordinate expression of the 2 gene sets.

Clinical outcome analysis. Tissue microarray data and clinical information were used for analyses (Supplemental Table 1). The predictive value of age, sex, stage, TNM status, differentiation status, 
and SETD2 expression univariately with relapse-free survival was analyzed using the Cox proportional hazards regression model. The HRs and CIs (95\% CI) were computed. Kaplan-Meier analysis for the relapse-free survival difference of the 2 cancer patient clusters was conducted using SPSS software. Multivariate Cox proportional hazards model analysis with the factors stated above was used to estimate the coefficients of the individual factor.

Accession codes. RNA-Seq and ChIP-Seq data were deposited in the NCBI's GEO (GSE95664).

Statistics. All experiments were performed using 3 to 15 mice or 3 independent repeated experiments from cells. Unless otherwise indicated, data in the figures are presented as mean $\pm \mathrm{SEM}$, and statistical significance was determined by 2-tailed Student's $t$ test. Cox proportional hazards regression model and multivariate Cox proportional hazards model analysis were performed with statistical software SPSS 22.0. Statistical significance was determined by 2-tailed Student's $t$ test, 1-way ANOVA, Pearson's correlation coefficients test, Kruskal-Wallis, log-rank test, or Fisher's exact test. For computing gene signature scores based on expression profiling data from human tumors, genes were first $z$-normalized to SD from the median across the primary tumor samples, and the average of the $z$-normalized values for all the genes in the signature was used to represent the signature score for each sample profile. For all statistical tests, a $P$ value of less than 0.05 was considered significant.

Study approval. All animal experiments were performed in compliance with the NIH Guide for the Care and Use of Laboratory Animals (National Academies Press, 2011) and were approved by the Institutional Biomedical Research Ethics Committee of the Shanghai Institutes for Biological Sciences. CRC specimens and their corresponding paracarcinoma tissue were obtained from patients who underwent surgical treatment from Shanghai Tenth People's
Hospital. The study was approved by the ethics committee of the Shanghai Tenth People's Hospital (SHSY-IEC-pap-16-24). All cell lines used are available at ATCC.

\section{Author contributions}

JQ and HY conceived and designed the experimental approach and prepared the manuscript as senior authors. NL and HY designed and performed most of experiments. NL and MJ contributed to computational analysis for gene signature analysis and statistical analysis. DF and JP performed tissue microarray and pathology analyses. JR, YL, TQ, QP, and YH performed and $\mathrm{JH}, \mathrm{QL}$, and XW supervised a specific subset of experimental design and analysis

\section{Acknowledgments}

We thank Sophia Tsai and Ming-Jer Tsai for critical comments and $\mathrm{Li}$ Li for Setd 2 flox mice. This work was supported by grants from the National Natural Science Foundation of China (31471281 and 81422030 to JQ, 81502440 to MJ, 81671115 and 81330054 to QL), the Chinese Academy of Sciences (QYZDB-SSW-SMC052), the Strategic Priority Research Program of the Chinese Academy of Sciences (XDB19000000), and the National Key Basic Research Program (2016YFC0902202). JQ is a scholar of the National 1000 Young Talents Program of China.

Address correspondence to: Jun Qin, The Key Laboratory of Stem Cell Biology, CAS Center for Excellence in Molecular Cell Science, Institute of Health Science, Shanghai Institutes for Biological Sciences, Chinese Academy of Sciences, 320 Yue Yang Road, Shanghai 200031, China. Phone: 86.21.54923326; Email: qinjun@sibs.ac.cn.
1. Baylin SB, Jones PA. A decade of exploring the cancer epigenome - biological and translational implications. Nat Rev Cancer. 2011;11(10):726-734.

2. Mikkelsen TS, et al. Genome-wide maps of chromatin state in pluripotent and lineage-committed cells. Nature. 2007;448(7153):553-560.

3. Edmunds JW, Mahadevan LC, Clayton AL. Dynamic histone $\mathrm{H} 3$ methylation during gene induction: HYPB/Setd 2 mediates all H3K36 trimethylation. EMBO J. 2008;27(2):406-420.

4. Hu M, et al. Histone H3 lysine 36 methyltransferase Hypb/Setd 2 is required for embryonic vascular remodeling. Proc Natl Acad Sci U S A. 2010;107(7):2956-2961.

5. Wagner EJ, Carpenter PB. Understanding the language of Lys36 methylation at histone H3. Nat Rev Mol Cell Biol. 2012;13(2):115-126.

6. Park IY, et al. Dual chromatin and cytoskeletal remodeling by SETD2. Cell. 2016;166(4):950-962.

7. Li F, et al. The histone mark H3K36me3 regulates human DNA mismatch repair through its interaction with MutS $\alpha$. Cell. 2013;153(3):590-600.

8. Pfister SX, et al. Inhibiting WEE1 selectively kills histone H3K36me3-deficient cancers by dNTP starvation. Cancer Cell. 2015;28(5):557-568.

9. Neri F, et al. Intragenic DNA methylation prevents spurious transcription initiation. Nature. 2017;543(7643):72-77.

10. Simon JM, et al. Variation in chromatin accessibility in human kidney cancer links H3K36 methyl- transferase loss with widespread RNA processing defects. Genome Res. 2014;24(2):241-250.

11. Gómez Acuña LI, Fiszbein A, Alló M, Schor IE, Kornblihtt AR. Connections between chromatin signatures and splicing. Wiley Interdiscip Rev RNA. 2013;4(1):77-91.

12. Zhu K, et al. SPOP-containing complex regulates SETD2 stability and H3K36me3-coupled alternative splicing. Nucleic Acids Res. 2017;45(1):92-105

13. Guo R, et al. BS69/ZMYND11 reads and connects histone H3.3 lysine 36 trimethylation-decorated chromatin to regulated pre-mRNA processing. Mol Cell. 2014;56(2):298-310.

14. Luco RF, Pan Q, Tominaga K, Blencowe BJ, Pereira-Smith OM, Misteli T. Regulation of alternative splicing by histone modifications. Science. 2010;327(5968):996-1000.

15. Dalgliesh GL, et al. Systematic sequencing of renal carcinoma reveals inactivation of histone modifying genes. Nature. 2010;463(7279):360-363.

16. Al Sarakbi W, Sasi W, Jiang WG, Roberts T, Newbold RF, Mokbel K. The mRNA expression of SETD2 in human breast cancer: correlation with clinico-pathological parameters. $B M C$ Cancer. 2009;9:290.

17. Fontebasso AM, et al. Mutations in SETD2 and genes affecting histone H3K36 methylation target hemispheric high-grade gliomas. Acta Neuropathol. 2013;125(5):659-669.

18. Huang KK, et al. SETD2 histone modifier loss in aggressive GI stromal tumours. Gut. 2016;65(12):1960-1972.

19. Zhu X, et al. Identification of functional cooperative mutations of SETD2 in human acute leukemia. Nat Genet. 2014;46(3):287-293.

20. Lao VV, Grady WM. Epigenetics and colorectal cancer. Nat Rev Gastroenterol Hepatol. 2011;8(12):686-700.

21. Liddle RA, Jirtle RL. Epigenetic silencing of genes in human colon cancer. Gastroenterology. 2006;131(3):960-962.

22. Fearon ER. Molecular genetics of colorectal cancer. Annu Rev Pathol. 2011;6:479-507.

23. Clevers H, Loh KM, Nusse R. Stem cell signaling. An integral program for tissue renewal and regeneration: Wnt signaling and stem cell control. Science. 2014;346(6205):1248012.

24. Schepers AG, et al. Lineage tracing reveals Lgr5+ stem cell activity in mouse intestinal adenomas. Science. 2012;337(6095):730-735.

25 . Vermeulen L, et al. Wnt activity defines colon cancer stem cells and is regulated by the microenvironment. Nat Cell Biol. 2010;12(5):468-476.

26. Barry ER, et al. Restriction of intestinal stem cell expansion and the regenerative response by YAP. Nature. 2013;493(7430):106-110.

27. Shen S, et al. rMATS: robust and flexible detection of differential alternative splicing from replicate RNA-Seq data. Proc Natl Acad Sci US A. 2014;111(51):E5593-E5601. 
28. Braunschweig U, Gueroussov S, Plocik AM, Graveley BR, Blencowe BJ. Dynamic integration of splicing within gene regulatory pathways. Cell. 2013;152(6):1252-1269.

29. Naftelberg S, Schor IE, Ast G, Kornblihtt AR. Regulation of alternative splicing through coupling with transcription and chromatin structure. Annu Rev Biochem. 2015;84:165-198.

30. Luco RF, Allo M, Schor IE, Kornblihtt AR, Misteli T. Epigenetics in alternative pre-mRNA splicing. Cell. 2011;144(1):16-26.

31. de la Mata M, et al. A slow RNA polymerase II affects alternative splicing in vivo. Mol Cell. 2003;12(2):525-532.

32. Itoh K, Brott BK, Bae GU, Ratcliffe MJ, Sokol SY. Nuclear localization is required for Dishevelled function in Wnt/beta-catenin signaling. J Biol. 2005;4(1):3.

33. Gan XQ, Wang JY, Xi Y, Wu ZL, Li YP, Li L. Nuclear Dvl, c-Jun, beta-catenin, and TCF form a complex leading to stabilization of beta-catenin-TCF interaction. JCell Biol. 2008;180(6):1087-1100.

34. Wang $W$, et al. FOXKs promote Wnt/ $\beta$-catenin signaling by translocating DVL into the nucleus. Dev Cell. 2015;32(6):707-718.

35. Kole R, Krainer AR, Altman S. RNA therapeutics: beyond RNA interference and antisense oligonucleotides. Nat Rev Drug Discov. 2012;11(2):125-140.

36. Mah AT, Yan KS, Kuo CJ. Wnt pathway regulation of intestinal stem cells. J Physiol (Lond). 2016;594(17):4837-4847.

37. Zhang Y, et al. H3K36 histone methyltransferase Setd 2 is required for murine embryonic stem cell differentiation toward endoderm. Cell Rep. 2014;8(6):1989-2002.

38. Fang D, et al. The histone H3.3K36M mutation reprograms the epigenome of chondroblastomas. Science. 2016;352(6291):1344-1348.

39. Lu C, et al. Histone H3K36 mutations promote sarcomagenesis through altered histone methylation landscape. Science. 2016;352(6287):844-849.

40. Ordóñez-Morán P, Dafflon C, Imajo M, Nishida E, Huelsken J. HOXA5 Counteracts stem cell traits by inhibiting Wnt signaling in colorectal cancer. Cancer Cell. 2015;28(6):815-829.

41. Dominguez-Brauer C, et al. Mule regulates the intestinal stem cell niche via the Wnt pathway and targets EphB3 for proteasomal and lysosomal degradation. Cell Stem Cell. 2016;19(2):205-216.
42. Lapierre M, et al. RIP140 increases APC expression and controls intestinal homeostasis and tumorigenesis. JClin Invest. 2014;124(5):1899-1913.

43. Freeman TJ, et al. Smad4-mediated signaling inhibits intestinal neoplasia by inhibiting expression of $\beta$-catenin. Gastroenterology. 2012;142(3):562-571.e2.

44. Dominguez-Brauer C, et al. E3 ubiquitin ligase Mule targets $\beta$-catenin under conditions of hyperactive Wnt signaling. Proc Natl Acad Sci U S A. 2017;114(7):E1148-E1157.

45. Voloshanenko $\mathrm{O}$, et al. Wnt secretion is required to maintain high levels of Wnt activity in colon cancer cells. Nat Commun. 2013;4:2610.

46. Walter DM, et al. Systematic in vivo inactivation of chromatin-regulating enzymes identifies Setd 2 as a potent tumor suppressor in lung adenocarcinoma. Cancer Res. 2017;77(7):1719-1729.

47. Pan W, et al. Wnt3a-mediated formation of phosphatidylinositol 4,5-bisphosphate regulates LRP6 phosphorylation. Science. 2008;321(5894):1350-1353.

48. Bilic J, et al. Wnt induces LRP6 signalosomes and promotes dishevelled-dependent LRP6 phosphorylation. Science. 2007;316(5831):1619-1622

49. Hamblet NS, et al. Dishevelled 2 is essential for cardiac outflow tract development, somite segmentation and neural tube closure. Development. 2002;129(24):5827-5838.

50. Etheridge SL, et al. Murine dishevelled 3 functions in redundant pathways with dishevelled 1 and 2 in normal cardiac outflow tract, cochlea, and neural tube development. PLoS Genet. 2008;4(11):e1000259.

51. Metcalfe C, et al. Dvl2 promotes intestinal length and neoplasia in the ApcMin mouse model for colorectal cancer. Cancer Res. 2010;70(16):6629-6638.

52. Serysheva $\mathrm{E}$, et al. Wnk kinases are positive regulators of canonical Wnt/ $\beta$-catenin signalling. EMBO Rep. 2013;14(8):718-725.

53. Spicer J, Rayter S, Young N, Elliott R, Ashworth A Smith D. Regulation of the Wnt signalling component PAR1A by the Peutz-Jeghers syndrome kinase LKB1. Oncogene. 2003;22(30):4752-4756.

54. Bonnans C, et al. Essential requirement for $\beta$-arrestin2 in mouse intestinal tumors with elevated Wnt signaling. Proc Natl Acad Sci U S A. 2012;109(8):3047-3052.
55. Chang CJ, et al. EZH2 promotes expansion of breast tumor initiating cells through activation of RAF1- $\beta$-catenin signaling. Cancer Cell. 2011;19(1):86-100.

56. Kouzarides T. Chromatin modifications and their function. Cell. 2007;128(4):693-705.

57. de Almeida SF, et al. Splicing enhances recruitment of methyltransferase HYPB/Setd 2 and methylation of histone H3 Lys36. Nat Struct Mol Biol. 2011;18(9):977-983.

58. Schwartz S, Meshorer E, Ast G. Chromatin organization marks exon-intron structure. Nat Struct Mol Biol. 2009;16(9):990-995.

59. Kolasinska-Zwierz P, Down T, Latorre I, Liu T, Liu XS, Ahringer J. Differential chromatin marking of introns and expressed exons by H3K36me3. Nat Genet. 2009;41(3):376-381.

60. Jung $\mathrm{H}$, et al. Intron retention is a widespread mechanism of tumor-suppressor inactivation. Nat Genet. 2015;47(11):1242-1248.

61. McGlincy NJ, Smith CW. Alternative splicing resulting in nonsense-mediated mRNA decay: what is the meaning of nonsense? Trends Biochem Sci. 2008;33(8):385-393.

62. Li N, et al. AKT-mediated stabilization of histone methyltransferase WHSC1 promotes prostate cancer metastasis. JClin Invest. 2017;127(4):1284-1302.

63. Kim D, Pertea G, Trapnell C, Pimentel H, Kelley $\mathrm{R}$, Salzberg SL. TopHat2: accurate alignment of transcriptomes in the presence of insertions, deletions and gene fusions. Genome Biol. 2013;14(4):R36

64. Kosinski C, et al. Gene expression patterns of human colon tops and basal crypts and BMP antagonists as intestinal stem cell niche factors. Proc Natl Acad Sci U S A . 2007;104(39):15418-15423.

65. Grimm C, et al. DNA-methylome analysis of mouse intestinal adenoma identifies a tumour-specific signature that is partly conserved in human colon cancer. PLoS Genet. 2013;9(2):e1003250.

66. Fevr T, Robine S, Louvard D, Huelsken J. Wnt/ beta-catenin is essential for intestinal homeostasis and maintenance of intestinal stem cells. Mol Cell Biol. 2007;27(21):7551-7559.

67. Qin J, et al. COUP-TFII inhibits TGF- $\beta$-induced growth barrier to promote prostate tumorigenesis. Nature. 2013;493(7431):236-240. 